\title{
A MULTIPLE WORKING MODE APPROACH TO HAMMERING WITH A MODULAR RECONFIGURABLE ROBOT
}

\author{
by \\ Vladyslav Romanyuk \\ Bachelor of Engineering, Ryerson University (2017) \\ A thesis \\ presented to Ryerson University \\ in partial fulfillment of the \\ requirements for the degree of \\ Master of Applied Science \\ in the Program of \\ Aerospace Engineering
}

Toronto, Ontario, Canada, 2019

C) Vladyslav Romanyuk, 2019 


\section{AUTHOR'S DECLARATION FOR ELECTRONIC SUBMISSION}

\section{OF A PROJECT}

I hereby declare that I am the sole author of this thesis. This is a true copy of the thesis, including any required final revisions, as accepted by my examiners.

I authorize Ryerson University to lend this thesis to other institutions or individuals for the purpose of scholarly research.

I further authorize Ryerson University to reproduce this thesis by photocopying or by other means, in total or in part, at the request of other institutions or individuals for the purpose of scholarly research.

I understand that my thesis may be made electronically available to the public. 


\title{
A MULTIPLE WORKING MODE APPROACH TO HAMMERING WITH A MODULAR RECONFIGURABLE ROBOT
}

\author{
Vladyslav Romanyuk
}

Master of Applied Science, Aerospace Engineering, Ryerson University, Toronto (2019)

\section{ABSTRACT}

Robot joints could be damaged by impulsive forces due to impact when the robot performs hammering operations. This thesis presents the development of a strategy that allows a modular reconfigurable robot to safely perform nail hammering. To do this, a multiple working mode approach is applied to switch the selected joint(s) to passive mode with friction compensation to allow free rotation during impact. Analytic impulse models are used to predict joint impulses which can be computed offline or online and serve as criteria for mode switching. Joint impulses are constrained in case the hammer collides with such an object of infinite effective mass. Advantages of the proposed approach may include savings on space, weight, costs, and complexity for a limited range of nail/board environments. An experimental study validates analytic models of hammering and effectiveness of multiple working mode approach. 


\section{ACKNOWLEDGEMENTS}

I would like to thank everyone in the Systems and Controls Lab for their support and curiosity. A thank you is to my supervisor, Dr. Guangjun Liu, who scouted me very early in my undergraduate studies without whom I could not finish at such a pace. Dr. Liu's willingness to let me take control of the work and bring it where I wanted is a special quality that I really appreciate. A special thanks also to Taras Pankov who offered many hours of his time to teach and train me. Thank you to the committee and staff at the Department of Aerospace Engineering who have provided exceptional support to my studies.

My family continually supported me throughout my studies to whom I am infinitely grateful for their trust and patience with me. 


\section{TABLE OF CONTENTS}

Author's Declaration for Electronic Submission of a Project ............................................ii

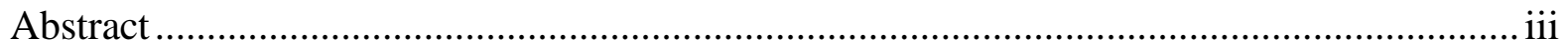

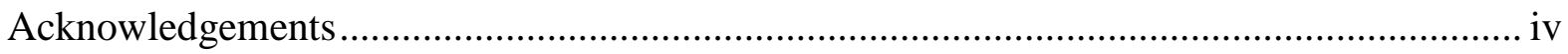

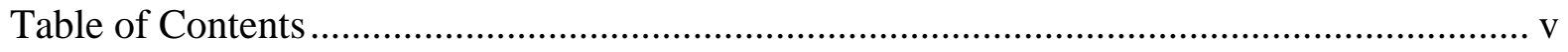

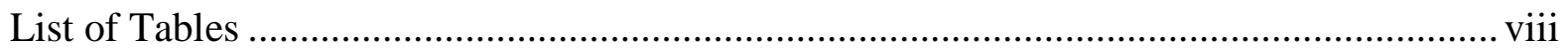

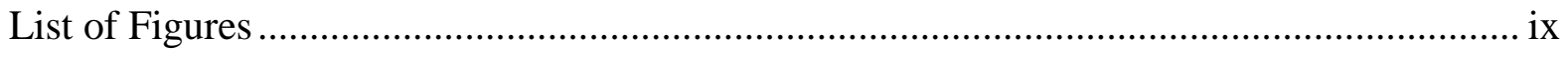

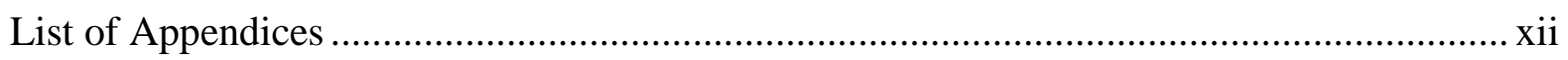

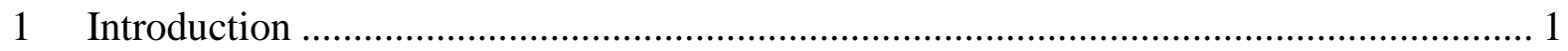

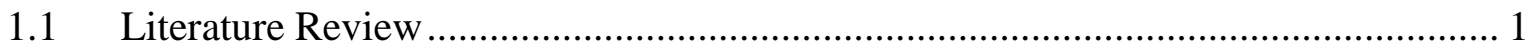

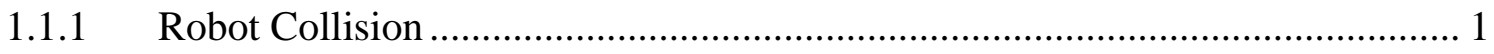

1.1.2 Prevention and Response Tasks (PRTs) ................................................. 3

1.1.3 Coordinated Striking Tasks (CSTs) …................................................ 7

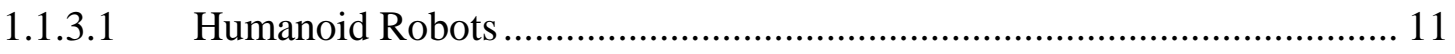

1.1.3.2 Variable Impedance Actuators ............................................................ 14 
1.2 Objectives and Contributions ………………………....................................... 20

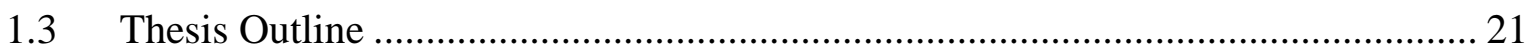

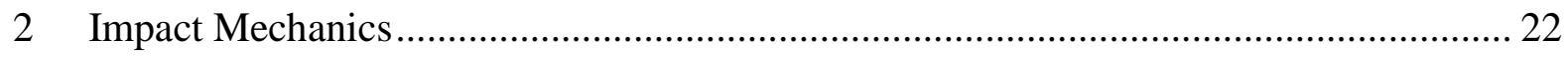

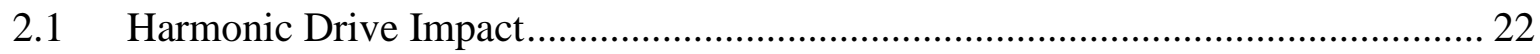

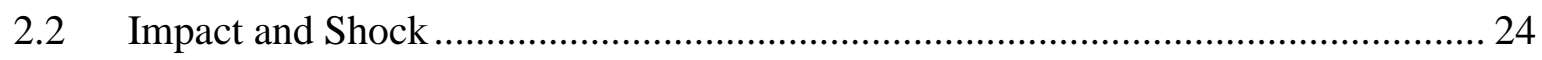

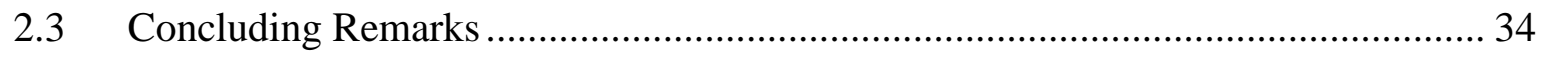

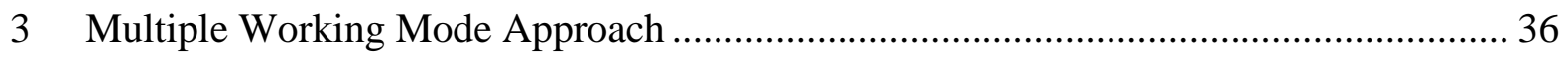

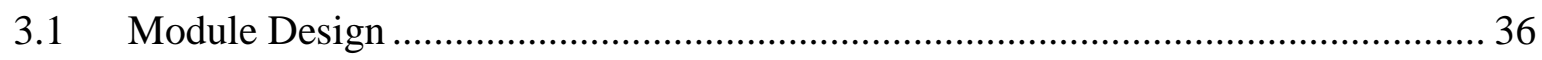

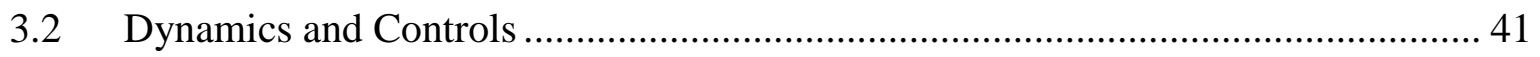

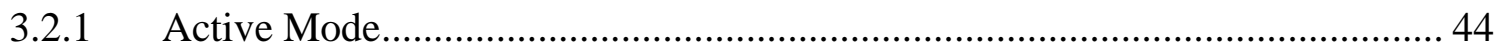

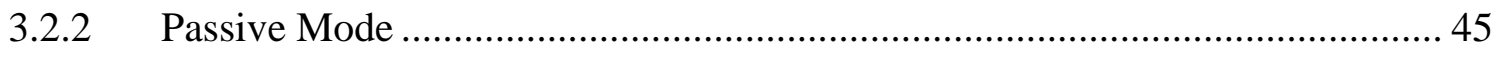

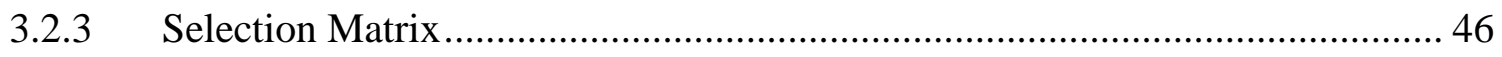

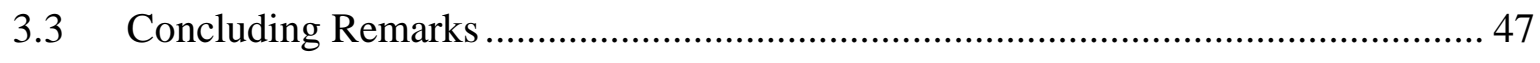

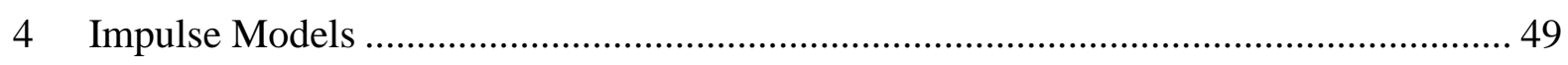


4.1 External Impulse Model ...................................................................... 49

4.2 Internal Impulse Model ......................................................................... 52

4.3 Concluding Remarks ................................................................................... 58

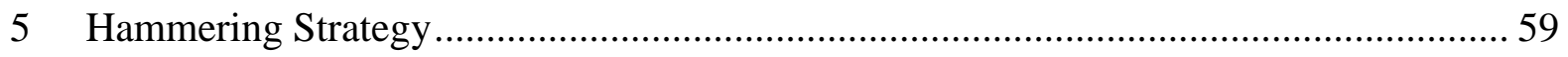

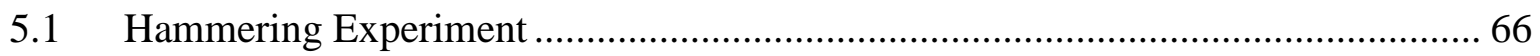

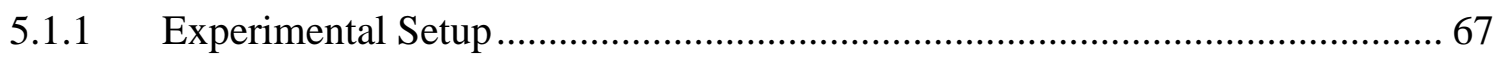

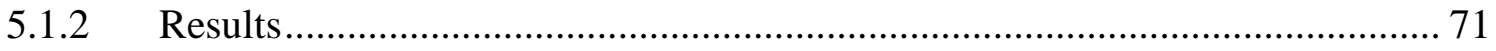

5.1.2.1 External Impulse and Effective Mass ............................................ 71

5.1.2.2 Internal Impulse and Passive Mode ................................................. 79

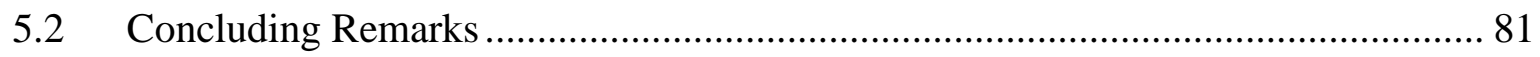

6 Conclusions and Future Works....................................................................... 82

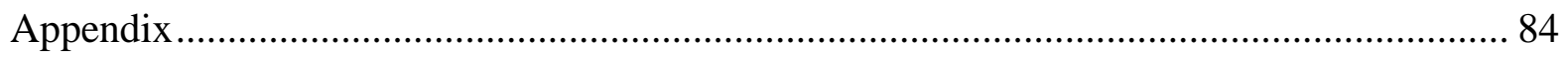

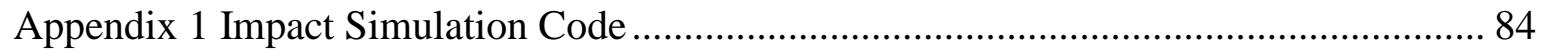

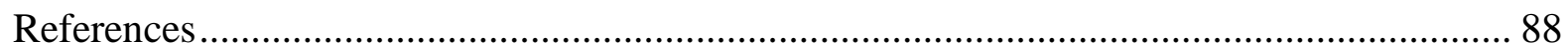




\section{LIST OF TABLES}

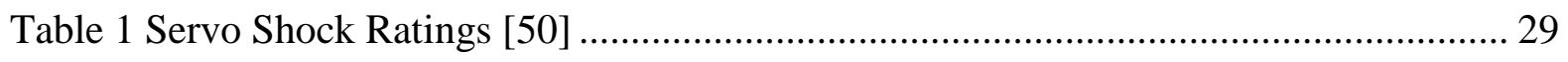

Table 2 External Torque for MRR module in passive Mode [47] ......................................... 41

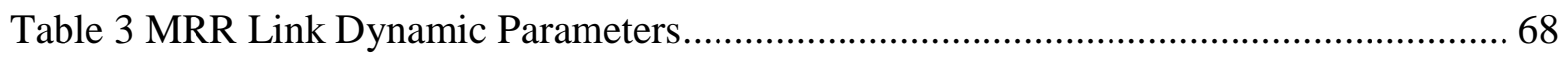

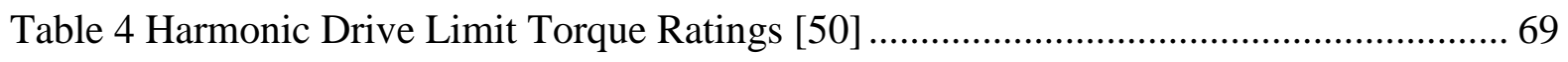

Table 5 Collision simulation results (unadjusted) ……………………............................. 71

Table 6 External F/T sensor styrofoam test 1 results.......................................................... 73

Table 7 External F/T sensor Styrofoam test 2 results ........................................................ 74

Table 8 External F/T sensor hardwood test results .............................................................. 75 


\section{LIST OF FIGURES}

Figure 1 Harmonic Drive Components [50]

Figure 2 Harmonic Drive S-N Curve [50]

Figure 3 Illustration of Principle of Impulse and Momentum on a Particle [48] 25

Figure 4 Two Particles with Initial Momenta Before Impact [48] 26

Figure 5 Collision Model of Two Particles [48] 26

Figure 6 Shock Resistance Specification Test [51] 30

Figure 7 Environment SRS (positive and Negative Spectra) [51] 31

Figure 8 Dimensionless Terminal Peak Sawtooth SRS scaled with the Amplitude and Frequency of the SRS [51]

Figure 9 Values of Dimensionless Frequency Corresponding to the First Passage of the SRS by the Amplitude Unit [51] 32

Figure 10 Simple (1) and Complex (2) Shock Transformations using the same

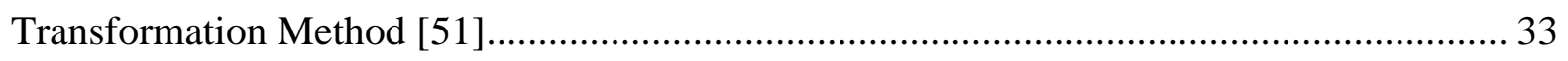

Figure 11 MRR Module Schematic [47] 37

Figure 12 Hardware Architecture [47] 37 
Figure 13 Control Architecture Layering [47]....

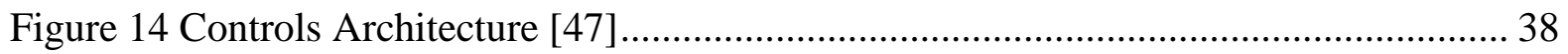

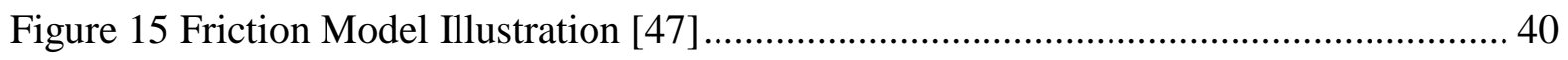

Figure 16 Hammer and Nail Environment Model [39] .................................................... 50

Figure 17 Force/Torque Model of a Serial Chain Robot [57] .............................................. 53

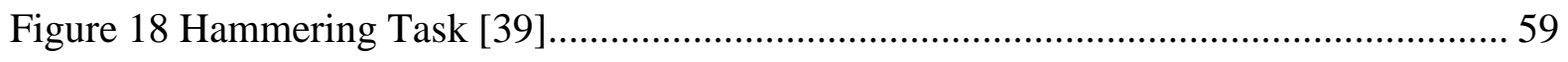

Figure 19 Harmonic Drive Limit Torques and Mode Switching............................................ 64

Figure 20 Diagram of MRR with Hammer End-Effector ........................................................ 68

Figure 21 Nail/Board Environment and F/T Sensor Set-up.................................................. 70

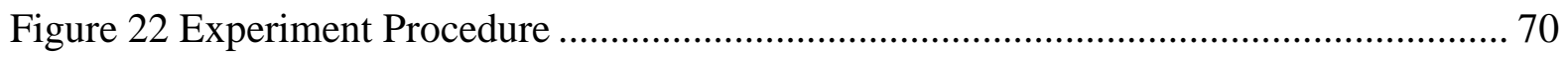

Figure 23 External force data for a styrofoam test ……………...................................... 72

Figure 24 External force data for a hardwood test........................................................... 72

Figure 25 Joint 2 position error during hardwood test (active mode).................................... 76

Figure 26 Test and simulation external impulse comparison .............................................. 78

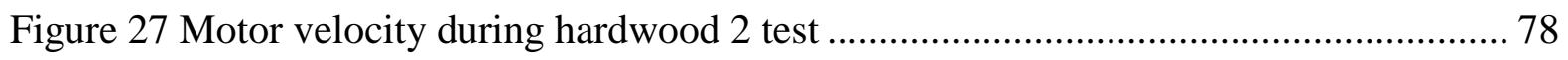


Figure 28 Frictionless joint velocity as compared to test values .. 


\section{LIST OF APPENDICES}

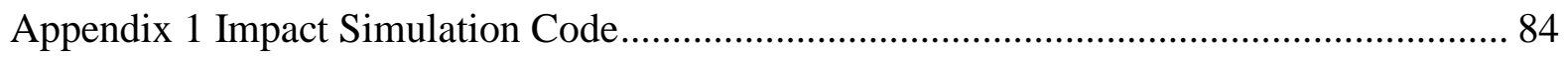




\section{INTRODUCTION}

The following section is aimed at providing the necessary background information to place the thesis within the context of research being conducted in the field of robot collision and impacts. The objective and contributions are presented at the end of the chapter along with the outline of the thesis.

\subsection{Literature Review}

\subsubsection{Robot Collision}

The application of robot manipulators in environments such as an industrial, space, and households quickly revealed the need to deal with a variety of collision related problems. One of the integral parts of a robot's capabilities relates to path planning and collision avoidance. While some of these applications are best solved by artificial intelligence and computer vision [1], many researchers agree that prevention is not enough and a study of the dynamics problems that arise during collision is necessary [2] [3] [4] [5] [6].

Going from household to space applications, however, presents a large spectrum of requirements and constraints for a fully generalized approach, so, generally, the methods have been tailored around a specific task for a given robot and available infrastructure. Vogel et al. [7] classified collision and impact tasks into two broad categories to distinguish their work from what is usually conceived of when discussing collision: 
1. Avoidance of collision or impact, and attenuation of energy transfer to or from the environment during unavoidable collisions and interactions (PRTs)

2. Hitting tasks for purposeful collisions and maximization of energy transfer (CSTs)

To avoid calling them "type 1 " or "type 2 " tasks, these categories are named in this work based on their distinctive feature - the intent behind the action. The first category is named "Prevention and Response Tasks" or PRTs while the second will be "Coordinated Striking Tasks" or CSTs.

As noted earlier, when thinking about collisions, most will consider the study of PRTs which includes research into object detumbling in space, humanoid robots in a home environment, as well as detection, handling, and reaction of industrial robots due to an accident [2] [3] [5] [6] [8] [9]. The study of CSTs, on the other hand, includes tasks such as kicking, hammering, and batting with humanoid or industrial robot arms [7] [10] [11] [12] [13] [14].

A different branch of research on impedance control [15] and on Series Elastic Actuators (SEAs) [16] emerged in over a decade ago [4] [14] [17] [18] [19]. Conventionally, mechanical impedance parameters of a joint are adjusted using a controller, but this concept introduced an elastic element with constant stiffness between the gear and the actuator output [19]. The concept was augmented in different ways towards Variable Impedance Actuators (VIAs) which included variable stiffness and damping elements; a subcategory of VIAs is called Variable Stiffness Actuators (VSAs) with only a stiffness element [19]. 
The successful application of VSAs to some collision problems [19] marks the question of whether it is valuable to investigate task-specific approaches. There is also the question of whether VSAs are mutually exclusive to that kind of research. To provide answers, a more detailed view into the kinds of problems these methods are aimed to solve, their advantages, disadvantages, and effectiveness is further studied.

\subsubsection{Prevention and Response Tasks (PRTs)}

The following section aims to highlight the key assumptions and features of a robot intended for a PRT in various environments and to narrow in on the direction of PRT research.

Consider the task of capturing a free-floating object in space by a robot arm mounted on a flexible structure or satellite like in [8]. The task can be split into three phases which include the pre-impact, impact, and post-impact phase with each phase having different challenges depending on the kind of assumptions made about the robot, the supporting structure, and the nature of the impact. Some of these important assumptions include:

- Serial, rigid-body system dynamics

- End-effector synchronization with the tumbling object is not possible even with visual sensing

- Conservation of momentum in the post-impact phase due to external force generated by collision 
Because of these assumptions, it is possible to take advantage of the fact that the partial momenta of the base and arm are dependent on the pre-impact configuration of the robot. Thus, there will be a configuration which minimizes the change of the base's partial momentum, but conversely maximizes it for the arm. High amplitude vibration of the flexible support structure may be induced due to momentum transfer to the base. The goal is to minimize the changes in the base momentum because any changes in velocity (especially angular velocity) of the base are much more difficult to handle than for the arm in space. This finding is important because it shows that pre-impact reconfiguration can be exploited for other collision tasks like hammering where the configuration before impact is determined by the user.

The work reported in [3] provides a glimpse into the direction of most PRT research. Some important aspects that were cited are safety issues in the operation of manipulators in unstructured workspace environments shared in close proximity with humans. Just as in [8], the authors investigate the challenges related to the three phases of an impact (pre-impact, impact, post-impact). Obviously, the intent is to avoid impact, so in the analysis of the preimpact phase there is an emphasis on avoidance.

The focus, however, is in developing strategies in the post-impact phase which include detection and reaction of the impact. An additional criterion of consideration was the cost effectiveness of proposed approaches. If possible, collision detection should be achieved without additional sensors to minimize cost. The main idea for sensor-less detection of collision is from [20], where collisions are viewed as faulty behaviours of the robot actuating 
system while the design of a detector takes advantage of the decoupling property of robot generalized momentum [3]. Further development is preceded by an analysis of the dynamics, energy, and momentum equations of an open kinematic chain, rigid-link, rigid-joint robot with an extension to variable-stiffness joint dynamics.

The work discussed thus far looked at collisions which are relatively tame in terms of speeds and severity. The sensing and processing apparatuses involved, such as encoders, torque sensors, visual sensors, and microprocessors, are not so highly stressed. [9] researched the capabilities of a DLR-LWR-III arm in the task of ball-catching, demanding a tight interplay of skills in mechanics, control, planning, and visual sensing. An active vision system for ball catching was used in the pioneering work on the 4-DOF WAM arm by [21], where a ball travelling at a velocity of 3-6 m/s was to be grasped by an arm within a 0.5 second window of time. The catching success rate was between 75-80\%. It was reported that the visual sensor accuracy was responsible for much of the failed trials [21].

While the parameters of the experiment in [21] have remained as a standard, only a $>80 \%$ success chance of catching a ball is reported in [9]. Due to the scaling of computational power, however, new strategies have been developed allowing even dextrous multi-finger hands to achieve these results. The hand can also make decisions, within such a short timeframe, between the types of grasp that would be most efficient. The speed required to achieve those results is a function of the design of the system architecture responsible for the computational resources of the robot. The DLR is equipped with two PCs running Linux, a cluster of $32 \mathrm{CPU}$ cores (responsible for motion generation), and a QNX real-time Robot 
Control operating system. Put together, this architecture can guarantee certain key speeds to perform inverse kinematics, trajectory generation, buffering, sending, and receiving of data [9]. The significance of this work is that it demonstrates the amount of computational power and hardware required to perform the ball-catching task with a multi-fingered hand. The cost is a potential drawback and it should not be ignored in future research projects.

Another popular path of research [6] [22] [23] [24] studies flexible robot structures using a finite element approach. In [6], the aim is to address issues during impact of lightweight flexible multibody operations which affect the vibrational characteristics of the mechanical system. Accurate models of flexible link-joint dynamics with impact are rarely studied because of their high complexity. The proposed method considers flexible links modelled as Euler-Bernoulli beams with high-order approximation coupling terms and flexible joints as a linearly elastic torsional spring with a mass. A simulation of a 2-DOF planar manipulator impact aimed to illustrate the method shows the significance of joint flexibility and high-order geometric nonlinearity of link deformational on end-effector motion [6].

Unlike with variable stiffness actuators that have an embedded spring element, the joint flexibility here is an outcome of flexibility in shafts, bearings, transmission, and other soft components. The importance of this branch is that it demonstrates some interest in more complex modelling of impact dynamics, but what is notable is the type of complexity being explored. Instead of adding simple components to the robot which increase the total system complexity, the inherent complex characteristics of the materials and structures is the focus. 
The issue is that there exists a trade-off between the complexity of analysis and complexity of a system being analyzed which creates a bottleneck for this line of work, which is why it hasn't found many applications.

Several important branches which aim to address the ability of manipulators to handle impacts in the sense of prevention and response have been discussed in this section. Some key assumptions and focus points of each branch and their effectiveness were summarized to give an insight on the direction of PRT research.

\subsubsection{Coordinated Striking Tasks (CSTs)}

The study of Prevention and Response Tasks has clear applications due to the unavoidable nature of collisions in various circumstances, so it attracted a lot of attention from different research groups around the globe with several pioneering works and inception points for different lines of research. The same cannot be said for the study of Coordinated Striking Tasks such as batting, hammering, and kicking which has become more popular only in recent years due to the development of VSAs and the desire for robots to work closely next to humans in a safe manner. There are, however, a few papers concentrating on very specific tasks like batting a baseball, kicking a soccer ball, tapping a shoulder with a small hammer, and humanoid robots striking objects with a rubber stick [7] [10] [11] [25] [26]. They share some similar features, but most are somewhat obscure in their reach.

Impacts of beam structures have been an interest for various structural engineers, but only a few groups have looked specifically into impact dynamics of robot links, much less 
intentional strikes and impacts [27] [28] [29] [30]. The initial work of [28] used a finiteelement model for impact analysis of a single-link flexible robotic arm and was incorporated into a variety of papers featuring flexible robotics.

A flexible link hammer for shoulder tapping, driving nails into boards at different angles, and control of a hammer using a neural network were proposed in [11] [25] [31]. Their main contribution was the development of a method to strike an object normal to its surface with a specific end-effector velocity. One issue that was found in the process is the fact that a flexible hammer will vibrate in the pre-impact and post-impact phase. The first mode of vibration may be utilized in the pre-impact phase, but the second and higher order terms need to be supressed since they can't be controlled. In the post-impact phase, all the modes of vibration must be supressed to prepare for the next strike. Not compensating vibrations successfully may result in diminishing the robot's ability to hit an object with a specified velocity in a given plane. Moreover, if the plane of motion includes gravitational forces, then the effect of vibrations is even larger. The proposed solution to suppress high order vibrations was an optimal regulator with a low-pass property, which was implemented and tested experimentally with success by driving multiple nails into a wooden board at different incidence angles [11].

An independent path of research funded by Adidas $A G$, the sports equipment company, studied and developed a humanoid robot leg for testing of soccer shoe and ball designs [10]. The action of kicking a ball during a professional soccer game produces a large impact on the leg which means that simulating the kick with a robot would stress the machine 
to its limits. Since several experiments were conducted where a ball would reach a takeoff speed of $40 \mathrm{~m} / \mathrm{sec}$, the study presents some interesting insights into the kind of design that would be selected for stressful striking tasks. After consideration of the limited data related to the biomechanics of kicking, the design converged to a passive energy-storage and freekick release system [10]. The dynamic analysis of a human leg kicking a ball was based on a simplified model of a translating double-pendulum system, which concentrated link-masses and lengths in proportion with that of a human leg [10]. The thigh rotation was actuated using a spring-loaded rotational actuator for a powerful swing, and a linear spring-loaded actuator was used to power the shank, which together could produce forces as high as $1000 \mathrm{~N}$. The experimental results demonstrate some failure of gear-meshes and overheating of motors during the initial phase of testing, but after some material hardening and motor re-winding the robot was able to perform 75 accurate kicks. After the delivery of the robot to the customer, the researchers proposed a maintenance contract for further development [10].

An important line of thinking starting with [32] built on top of very basic rigid body dynamics and collision theory in [33] to introduce methods of evaluation for serial and multiarm collisions. A number of measures, namely the dynamic impact ellipsoid and generalized impact ellipsoid, were developed as a means of finding optimal impact configurations based on an external impulse model of a robot end-effector during a collision with another rigid body. The dynamic impact ellipsoid corresponding to changes in external impulse with respect to changes in end-effector velocities can be used to evaluate potential damage to the end-effector in case of a collision. The generalized impact ellipsoid, on the other hand, was aimed at analyzing changes in external impulse with respect to changes in kinetic energy of 
the robot joints. Both measures are useful in cases where end-effector and environment damage and design are of importance [34]. However, by mapping the external impulse onto the internal impulses at robot joints, [34] was able to develop an internal impact measure that evaluated changes in internal impulse with respect to external impulse experienced at the end-effector. At the same time, [34] expanded the dynamics of a rigid serial and dual-arm robots to collisions with non-rigid surfaces through an effective mass model of the surface the robot end-effector would contact. The hardness of the surface is reflected in its effective mass such that a harder surface would cause a larger external force. The effective mass is estimated through testing by measuring the duration of impact and depth that a nail is driven into a board. This data provides the velocity at which the nail travels due to impact and combined with measurement of impulsive force magnitude using a F/T sensor, the effective mass of the nail/board system can be calculated. The effectiveness of the impulse models and impact measures was confirmed using simulation of various manipulator types and experiment using a closed-chain manipulator performing sawing and hammering tasks [32] [34] [35] [36]. A piezoresistive F/T sensor with a less than 5 microsecond response time was used for fast measurement of external impulsive forces/torques, but the internal impulsive forces were calculated using a model as a function of external impulse [35]. Joint torque sensors could potentially be used to corroborate the internal impulsive model further.

After the first prototypes of variable stiffness actuators in 2003 and a number of humanoid robot technologies [18], most of the research into Coordinated Striking tasks has been in the form of conference papers that aimed to apply these technologies and express their applicability to a wide range of tasks which included hammering and batting [7] [13] 
[26] [37] [38] [39]. The approaches which include VSAs and humanoid robots are discussed separately.

\subsubsection{Humanoid Robots}

The HOAP-2, HRP-2 and Hiro are humanoid robots that have some striking capabilities like performing the karate chop, hitting a nail into a wall, and playing the glockenspiel [26] [37] [38]. While the development of these capabilities remained in the form of single conference papers, each study has an experiment associated with it, which means it requires some attention and unpacking.

The authors of [37] had the HOAP-2 perform a karate chop with the intent of testing their method of performing an impact task while keeping the humanoid robot physically stable on its two feet. They tried to address several issues which included optimization of working postures, motion synthesis, stability analysis, and control of the robot in postimpact. The analysis of the karate chop postures and motions was inspired by the way a man would try to exert a large force in some motions which means it was designed heuristically by the authors [37]. To keep stability during the karate-chop motions, the authors used a ZMP (zero moment point) compensation control to track a desired ZMP by adjusting the horizontal motion of the torso combined with an inverted pendulum control method for dynamic balance of the torso [37]. Numerous experiments on three different materials (mainly balsa wood) were conducted to confirm the applicability of the method which resulted in an estimated average momentum required to break the board of 0.29 Ns (newtonseconds). The measurements were done by analyzing total force on the feet of the robot. A 
maximum velocity at the end-effector was estimated to be $1.77 \mathrm{~m} / \mathrm{s}$. The experiments also show small improvement in the convergence of the ZMP back to equilibrium point in postimpact due to the control methods [37]. The experiment opened some doors but left many questions unanswered - questions relating to optimal posture methodologies and automatic motion generation that isn't based on intuition as well as more in-depth analysis of postimpact control dynamics.

In 2008, [38], which included researchers from the HOAP-2 experiment, set out to develop a motion generation method in the HRP-2 humanoid robot that did not rely on intuition for driving a nail into balsa wood. The main idea behind the motion generation scheme is to maximize the nail driving depth through a choice of a proper objective function. It was shown experimentally that the magnitude of the integral of force history (related to impulse) is positively correlated to the nail driving depth. Therefore, if they can use the function to predict the impulse exerted during the movement, it should be possible to predict the driving depth as well. The prediction of impulse is done using the concept of virtual mass from [40]. As part of their three-step motion generation method (in pre-impact, impact, and post-impact motion), the joint positions and velocities that minimize the objective function under a set of constrain factors are solved using SQP (Sequential Quadratic Programming) method [38]. Given these positions and velocities, the joint trajectories are interpolated by quintic interpolation [38]. While the experiments show the successful performance of the hammering task, the model predictions were off from the actual penetration of the nail into the board ( $2 \mathrm{~mm}$ vs $11 \mathrm{~mm}$, respectively). The authors attribute this error to the fact that the 
dynamic model does not consider joint torques and equivalent inertias of the motor and reduction gears [38].

In recent years, the use of humanoid robots has broadened, and their application to entertainment and human cooperation purposes has begun to penetrate daily life [26]. A few robots that could perform drum beating performances using force or sound as feedback have been attempted in the past [26] [37]. The authors of [26] saw this as an opportunity to give Hiro, The Humanoid Robot the ability to perform on a glockenspiel, a percussion instrument composed of a set of tuned keys arranged like a keyboard, using a flexible rubber stick (RS) and stereo cameras (in his eyes) for visual and audio feedback. To make the robot both useful in entertainment and possibly industry, he is equipped with low-power servo motors and constrained to a limited work space. The impact task is controlled through a method called Input Shaping whereby an input command controls the object vibration by considering the natural frequency of vibration and its phase [26]. The method amplifies certain modes of vibration and reduces the ones that are harmful for the task by sending inputs from the robot arm that are in or out of phase with the vibration of the stick. Humans tend to use the wrist while playing the glockenspiel for snap motions, but snap motions in a robot could be quite damaging. The idea is to make the RS vibrate using the robot arm as an input and hit the keys of the instrument when the stick is at the bottom of its vibration cycle. Side-to-side motion also causes vibrations - these are undesirable and thus need to be reduced. Experiments where the robot performs key-to-key movements and strikes them with different input patterns were successful in demonstrating that the keys can be consistently pressed in a 
desired location within fractions of a second $(0.23 \mathrm{sec})$ while reducing the side-to-side vibrations amplitudes to values below 3mm [26].

The current state of research into coordinated striking tasks of humanoid robot shows that there are no major branches of research at the spear of the field, but rather that certain robots are getting better at doing specific tasks using interesting methodologies all the while ensuring that the methods fit into the current interest trends surrounding robots such as safe collaboration with humans and industrial applications.

\subsubsection{Variable Impedance Actuators}

The broadened landscapes of robotic applications to environments where they are interacting or cooperating with humans has led to a huge interest of studying robot safety. In a 2004 paper, [41] discuss important factors and trade-offs between safety and performance in robot arm design and controls. That year, the same authors filed a patent for the concept of Variable Impedance Actuators (VIAs) based on a prototype which uses two independently controlled brushless DC motors connected to the joint shaft by a timing belt, where the belt itself is tensioned by three idle pulleys and connected to the casing by passive elastic elements [42]. The DC motor positions can thus be used to control the joint stiffness during execution. This was only the first concept of VIAs which has since been overshadowed by other technologies such as the Floating Spring Joint (FSJ) [17] and the Bidirectional Antagonistic Variable Stiffness Joint (BAVS) [43] used in the extremely popular DLR Hand Arm System [4]. 
In general, the relationship between collision research and VSAs is that of application. In other words, collision-related tasks are viewed simply as a possible area to which VSAs can be applied. The VSA hammer, the hugging robot Probo, a one-legged hopping machine, a bipedal robot, and many other projects have been reviewed by Wolf et al. [19] in their 2016 study which is also a guide to the design and selection of VSA components based on a desired task. Because of this top-down approach, the research tends to be focused in developing solutions to problems related to the VSAs rather than problems related to the overall task. Consider the VSA hammer study where the problem that was being addressed is maximization of link speed for both constant and variable stiffness actuators through optimal control theory [13]. Based off the problem statement, the hammering task is merely an application which makes this a top-down approach toward a coordinated striking task. Instead of talking about the specific studies that mentioned VIAs/VSAs in relation to a CST, it is therefore easier to simply discuss the generic advantages and disadvantages that they bring to the table when equipped on a robot. The following are the most common use-cases of VSAs [19]:

1) Shock absorption;

2) Stiffness variation with constant load;

3) Stiffness variation at constant position;

4) Cycling movements; and

5) Explosive movements. 
Out of those five cases, a striking task by its nature of short duration would not require capabilities in 2) and 3) which are meant for interactions which require prolonged contact and friction. Shock absorption, cyclical movements, and explosive movements (high acceleration), however, are applicable to certain striking tasks.

The shock absorption capabilities of VIA come from the intrinsic passive flexibility which can react to fast and hard loads without any need for computation and control from the computer. A rigid link is connected directly to the gearbox (e.g. harmonic drive), but in a VIA the two are decoupled by a spring/damper unit which reduces the peak torques seen by the drive train and cushion the actuator from overload [19]. Industrial applications tend be very cyclical in the sense of repetition of the same task throughout the work day. This is not quite the same sense that [19] describes the advantages of VSA to cyclical movements. Here, cyclical movements are described as repetitive accelerations and decelerations of the robot where the VSA is modelled as a two-mass system with the link-mass being excited by a motor (the second mass) such that it oscillates [19]. In the deceleration phase of the oscillatory movement, the kinetic energy can be stored in the spring as elastic potential energy and once the maximum amplitude is reached, the stored energy can be released to accelerate the spring without the motor doing extra work [19]. This can be energy saving depending on how it's applied since the motor doesn't have to perform as much motion. An explosive movement is simply one in which the robot accelerates to a high velocity over a short period of time. To achieve a higher output peak velocity, the VSA spring can be preloaded by blocking the actuator output and abruptly released like when flicking a finger [19] [44]. Another possible way to increase the velocity is to use a strategy that was describe 
in the cyclic movements where by a spring is loaded for a half-cycle and with additional help of the motor can result in a similar wind-up [19].

The advantages of VSAs do not come without their disadvantages and there are considerable trade-offs that need weighing before making the choice of using VSA. The following is pointed out in [19]:

VSA are usually intended to be used in robotic arms and legs, where the possible size and weight are very limited. The units have to be very compact to fit in such a system, especially if it is intended to be a mobile system like a humanoid of adult or even smaller size. The weight of the VSA units is a dominant factor in these systems. So, the weight of the VSA directly affects the system performance because it statically reduces the payload in an environment with gravity and increases the inertia of the links, which reduces the active and passive bandwidth of the system. Compared to a common rigid robotic actuator, a VSA is a much more complex system. To be able to change the stiffness, each actuator has to consist of at least two motor units, usually with two corresponding gears, and a spring mechanism (see [45]). A rigid robotic joint has typically only one motor unit, one gear, and possibly a torque sensor. In VSA with no passive damping, usually the torque sensor can be omitted by using a good model of the spring characteristic. Nevertheless, a robotic joint equipped with a VSA is more expensive than a common robotic joint. The costs of a high performance commercial robot with VSA built in are likely to be more. Depending on the budget, the VSA has to be composed of cheaper and in most cases inferior parts. This will limit the capabilities of the whole robot. In total there are at least three characteristics of VSA which 
are added to the system and then traded against each other, depending on the constraints and task of the system - cost, weight, and size. Moreover, VSAs add complexity to the robot, so it is no wonder that so much research effort is being poured into the field when there is a diverse set of issue and applications which need to be addressed and optimized. However, as shown before, the types of issues being addressed are often related to the performance of the VSA itself.

Analyzing the history, applications, advantages, and disadvantages of VSAs shows that they are not treated as necessary components of a robot system, but rather like modules that can be used to boost and enhance some system capabilities if there is enough advantage to using the VSA for some generalized attribute of the robot. Obviously, there will be cases where applying VSA would be advantageous to the overall task execution, but the monetary costs or available space (physical or computational) is simply not enough which is why research into task-specific approaches with rigid robot dynamics is still valuable and complementary to VSA research. There will be newly developed ways to augment the robot in the future when funds or space become available with increasing developments in other fields like motor capacity and computation, so the multiple branches of research can easily run parallel to each other.

\subsubsection{Modular Reconfigurable Robots}

VIAs provide many advantages to a robot, but one must sacrifice in terms of weight, size, cost, and complexity. Depending on the application, these factors tend to be more

pronounced. For example, space applications which use Modular Reconfigurable Robots 
(MRR) tend to require compact and simple to assemble robot equipment such that it's easier to transport to space and to maintain/operate during a mission [45]. Industrial applications may not respect weight and size as much, but reductions in cost and complexity tend to be favored especially for scalability. This presents a special opportunity to develop a striking method for MRRs without the use of VIAs which would also make it the only method available specifically optimized for striking applications in space. If there is already a robot being used for space missions, the new method could also be crafted such that it does not require any extra changes to the hardware - a clever impact strategy combined with a software update with no hardware modifications needed.

The main idea behind VIAs can be split into two components: passivity and compliance. Recent work by Ahmad et al. [46] uses a multiple working mode approach (MWMA) for door-opening where each joint of a modular reconfigurable robot on a mobile platform can switch freely between active and passive modes. The rigid-link serial robot opens the door while preventing build-up of internal forces by setting some of the joints into passive mode during the door-opening [46]. Experiments have shown the method to be effective in reducing internal forces, comparable to other methods which apply complicated compliance techniques [46]. The MWMA [47] can be an invaluable tool since it brings the passivity portion of VIAs and allows the joints to switch between active and passive modes seamlessly. 


\subsection{Objectives and Contributions}

The objective of this research is to develop a method of striking a small object, or surface, with a hammer-like end-effector using a mobile Modular Reconfigurable Robot. A problem occurs when joints in position control experience impact. The motor does not have time to react and compensate the impulsive torques but even so, it attempts to bring the joint back to the reference position requested by the controller causing a joint torque build-up. This problem is the focus of this thesis. One requirement for this method is that it should not make changes to the robot hardware such that its dynamics are affected other than simply switching the end-effector. Because solid body impact dynamics is an extremely complex topic, beyond the aim of this research, a literature review was conducted to determine if current methods of impact mechanics are able to provide a working solution for the robot/environment interaction. The contributions of this work include:

- Integration of analytic impact models with the Multiple Working Mode Framework in a Modular and Reconfigurable Robot

- Joint selection criteria and criteria for real-time mode-switching

- Impulse models calculate potential damage through offline or online simulation, joints are selected based on the hardware limits

- Encoder measurements to time the switching from active to passive mode

- Procedure for conducting safe hammering operations that integrates the criteria

- Includes optimal striking configurations and velocities

- Experimental testing of impulse models and effectiveness of passive mode 


\subsection{Thesis Outline}

Details about impact and shock in harmonic drives are discussed in Chapter 2 with a focus on the interpretation of the mathematical models of impact and how they relate to body flexibility. Chapters 3 and 4 go through the derivation and integration of MRR dynamics and controls using multiple working mode framework with the external and internal impulse models. Chapter 5 is a primary contribution which presents the step-by-step strategy for hammering which includes the joint selection criteria and method of timing the mode switching. An experimental study of the strategy is also presented to see whether the joint torque sensors are able to measure impulsive forces due to collision to verify the impulse models. Chapter 6 concludes the thesis with a short summary, a list of advantages and limitations, and future work. 


\section{IMPACT MECHANICS}

An impact (or collision) between two solid bodies is characterized by its short time frame. What happens in this short time frame is an extremely complicated problem involving solid bodies and elasticity with a small set of currently applied solutions [33] [48] and very few upcoming alternatives [49]. This section of the thesis outlines the ideas and assumptions at the core of rigid body impacts by analyzing an interaction between two simple particles. It starts off by explaining how this theory is relevant to impacts experienced by a harmonic drive and hardware limitations. Shock theory and testing methods are presented with a discussion about why it is not a good alternative when applied to harmonic drives.

\subsection{Harmonic Drive Impact}

The conventional harmonic drive transmissions consist of three basic components including the Wave Generator (WG), Flexspline (FS), and Circular Spline (CS) seen in Figure 1. In robotic applications, harmonic drives are used as speed reducers that allow a small motor to drive the joint as compared to a direct drive joint where a large motor is necessary. In standard configuration for a robot joint, both the motor and the link act on a shaft running through the center of the wave generator where the flexspline is fixed. When the end-effector collides with the environment, its respective link twists the shaft and thus it also twists the wave generator. The flexspline and the wave generator bearing are very sensitive components of the harmonic drive. The life of the harmonic drive is determined by the life of the wave generator bearing under normal working condition which is labelled in 
the figure below, but because of the repeated deflection of the flexspline during collision, the torque capacity of the gear, determined by the strength of the flexspline, is the limiting factor [50]. If an excessive torque is applied to the gear, the teeth could disengage and cause Ratcheting. The impact torque (or impulsive torque, defined rigorously in the next subchapter) must be below the Momentary Peak Torque limit to ensure long-term gear integrity, but this exposure must be minimized since it reduces the overall life of the gear [50].

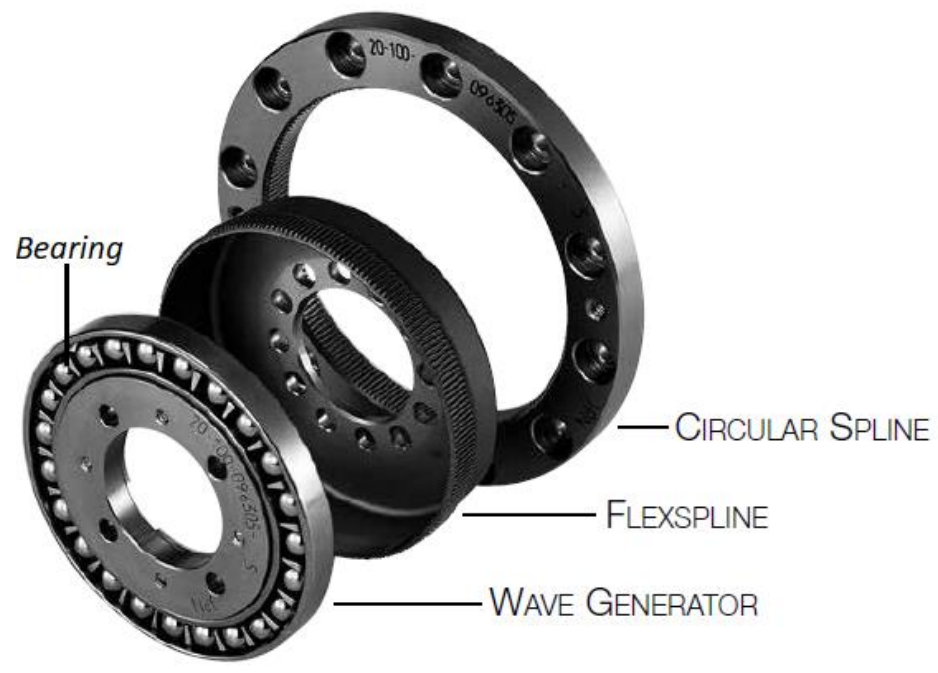

Figure 1 HARMONIC DRIVE COMPONENTS [50]

For safe repetitive action, the threshold below the Momentary Peak Torque, the Repeated Peak Torque, can be used as a limit to ensure the gear goes through its average fatigue life. If torque input from the link-side is below the Rated Torque values, the harmonic drive assembly will be operating safely. The three limit values can be seen in Figure 2 which is the S-N curve (Stress-Number of cycles) of the harmonic drive components normalized to the rated torque. The infinite fatigue life of a component is defined as $10^{7}$ input rotations, but 
if the aim is to operate more than that number, the wave generator curve shows a decrease in strength, but the flexspline stagnates.

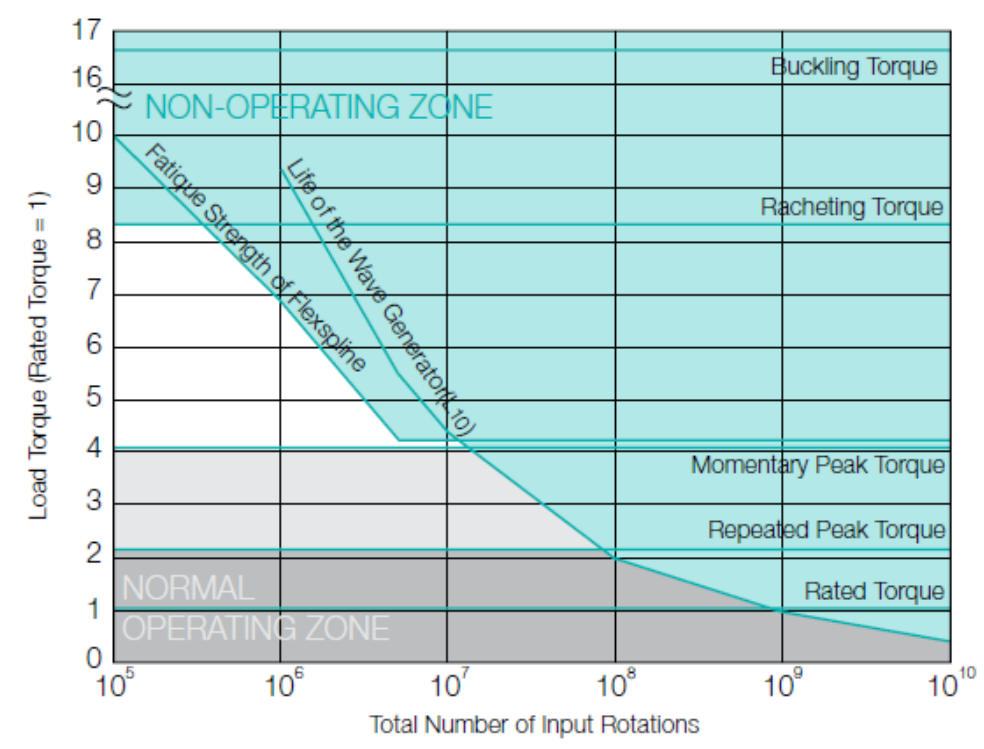

Figure 2 HaRmonic Drive S-N CURVE [50]

\subsection{Impact and Shock}

The aim of this chapter is to introduce the basics of impact modelling by the example of two particles with mass $m$ and negligible size and shape colliding. The same idea illustrated with this linear collision can be applied to a variety of impacts including hammering. To start the analysis, the terms relevant to the interaction between two solid bodies moving at some velocity as in Figure 3 and Figure 4 below are defined.

1. The integral $\sum_{j} \int_{t_{1}}^{t_{2}} \boldsymbol{F}_{j} d t$ is an impulse; 
2. A force, $\boldsymbol{F}_{\boldsymbol{j}}$, causing a negligible impulse is called non-impulsive force, and large forces acting over short time that change the particle's momentum significantly are called impulsive forces;

3. External forces change the velocity of a particle and are included in system momentum equations as an external impulse, while internal forces get cancelled out by their opposite and equal collinear counterpart and are not included;

4. To determine the internal impulses on a particle, the particle must be isolated from the rest of the system and the force must be treated as external.

The particle is assumed to be rigid throughout the application of impulse to its surface.

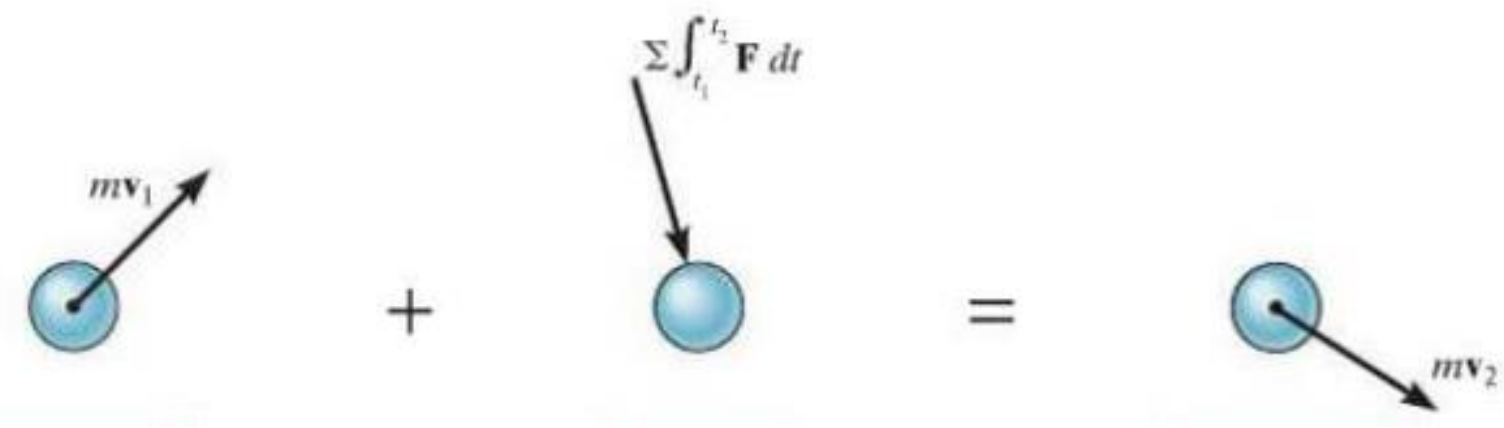

Figure 3 Illustration of Principle of Impulse and Momentum on a Particle [48]

Connecting these ideas closer to the hammering task, the impulsive force acting between the hammer and nail impact may be considered external (like in this work) to determine the effect on the hammer. Then, if the interest is in ensuring the integrity of the hammer tool, this force can be minimized. Internal impulses on each robot joint connected to the hammer may also be isolated in a similar fashion and their corresponding impulsive force 
must be resisted by the link and joint structural components. Susceptible components of a harmonic drive transmission must be accounted for as explained in Chapter 2.1.

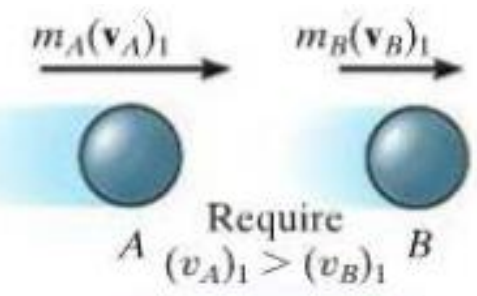

\section{Before impact}

\section{Figure 4 Two Particles with Initial Momenta Before Impact [48]}

The concept of the coefficient of restitution is the most common way to solve for the post-impact velocities of a multi-body collision. It is very important to understand the difference between the modelling and the physical reality of what happens when two particles like in Figure 4 collide. First, logic behind the classical development of impacts in [48] is presented and later its underlying assumptions are broken down further to get to the core of what the equations are saying about the interaction.

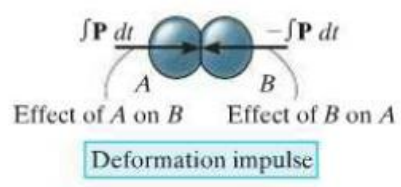

(a)

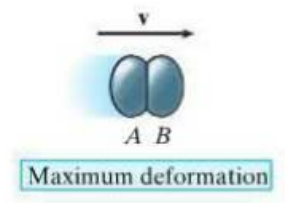

(b)

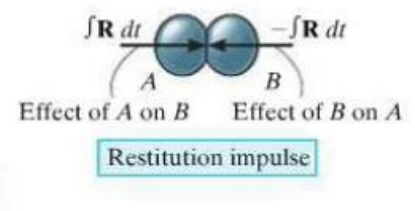

(c)

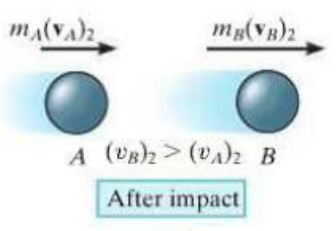

(d)

Figure 5 Collision Model of Two Particles [48] 
Figure 5 depicts two particles with initial velocities moving along a line such that eventually a collision will occur. During the first part of the interaction, the particles are treated as nonrigid and their surface is deformable. The bodies experience a linear deformation impulse, $\int \boldsymbol{P} d t$, as in Figure 5(a) until the maximum possible deformation is reached at which point the relative velocity of both particles is exactly zero and they are moving at the same velocity with respect to another frame of reference placed in the world. Next, the bodies experience a period of restitution where a linear restitution impulse, $\int \boldsymbol{R} d t$, as in Figure 5(c) acts on the bodies to push them apart. Finally, the interaction is complete when the two particles have the momenta defined by their post-impact velocities. The coefficient of restitution, $e=\frac{\int \boldsymbol{R} d t}{\int \boldsymbol{P} d t}$, is defined as the ratio between the restitution impulse and deformation impulse. Clearly, if the restitution impulse is zero then the two bodies are stuck together (plastic collision) and if the restitution impulse is equal to the deformation impulse, then the two bodies go their separate ways (elastic collision).

Now, while it sounds like the interaction is modelled in a way that accounts for the elasticity of the bodies and the rigid-body assumption is violated within this model, that is not the case. Consider the system comprising of just one rigid particle moving at some initial velocity which experiences two external forces, $\boldsymbol{P}$ and $\boldsymbol{R}$, over a short time like in Figure 3. The impulse due to $\boldsymbol{P}$ and $\boldsymbol{R}$ can be modelled as $\int F\left(m_{B}, v_{B}\right) d t$, where B is an imaginary particle. Equation (1) is the conservation of momentum for the collision of two particles, A and $\mathrm{B}$. 


$$
m_{A}\left(v_{A}\right)_{1}+m_{B}\left(v_{B}\right)_{1}=m_{A}\left(v_{A}\right)_{2}+m_{B}\left(v_{B}\right)_{2}
$$

Equation (2) is simply the linear momentum of particle A with two external impulses. Impulse $\int \boldsymbol{R} d t$ in (2) is eliminated from the equation by the relationship defined through the coefficient of restitution in (3).

$$
\begin{gathered}
m_{A}\left(v_{A}\right)_{1}-\int P d t-\int R d t=m_{A}\left(v_{A}\right)_{2} \\
\int R d t=e \cdot \int P d t
\end{gathered}
$$

Then, by substituting (3) into (2) and rearranging (1) as seen in equation (4), $\int F\left(m_{B}, v_{B}\right) d t$ can be defined:

$$
m_{A}\left(v_{A}\right)_{1}+\underbrace{m_{B}\left(v_{B 1}-v_{B 2}\right)}_{\int F\left(m_{B}, v_{B}\right) d t=-(1+e) \int P d t=m_{B}\left(v_{B 1}-v_{B 2}\right)}=m_{A}\left(v_{A}\right)_{2}
$$

The solution to this problem is simply that of applying the principle of linear momentum by doing a summation about an external frame of reference. The concept of coefficient of restitution thus represents a sort of black box based around the human intuition of what happens in such an interaction between spring-like particles. However, attempts at modelling what happens inside this black box within such a time frame have yet to be successfully applied to various engineering applications [49] due to complexity and the relative fidelity of 
this model. In most applications, simply testing the ability of the device to withstand impact force or shocks experimentally is enough since they are abnormal conditions. Harmonic drives and other transmission systems are most commonly tested for their impact strength; however, the following section discusses why shock theory and shock testing is not performed on harmonic drives.

Within the realm of harmonic drives, shocks and shock testing is not as common as with joint actuators and other small mounted devices. In relevant literature [51], a shock is defined as a vibratory excitation with a duration between one and two times the natural period of the excited mechanical system. More precisely, the excitation and sudden changes in acceleration of the system is what is most commonly considered when talking about shocks. The same term may less commonly refer to changes in force, velocity, or position, which is where the term shock impulse is derived. Note that while during an impact a body also experiences a shock, but when a body experiences a shock, it is not necessarily due to an impact (impacts require two colliding bodies in this terminology).

TABLE 1 Servo SHOCK RATINGS [50]

\begin{tabular}{|c|c|c|}
\hline \multicolumn{1}{|c|}{ Model } & Vibration Resistance $\left(\mathrm{m} / \mathrm{s}^{2}\right)$ & Shock Resistance $\left(\mathrm{m} / \mathrm{s}^{2}\right)$ \\
\hline FHA-C mini Series & 25 (frequency: 10 to $400 \mathrm{~Hz})$ & 300 \\
\hline FHA-C Series & 24.5 (frequency: 10 to $400 \mathrm{~Hz})$ & 294 \\
\hline $\begin{array}{c}\text { HA series Servo } \\
\text { Driver }\end{array}$ & 4.9 (frequency: 0 to $55 \mathrm{~Hz})$ & 98 \\
\hline
\end{tabular}




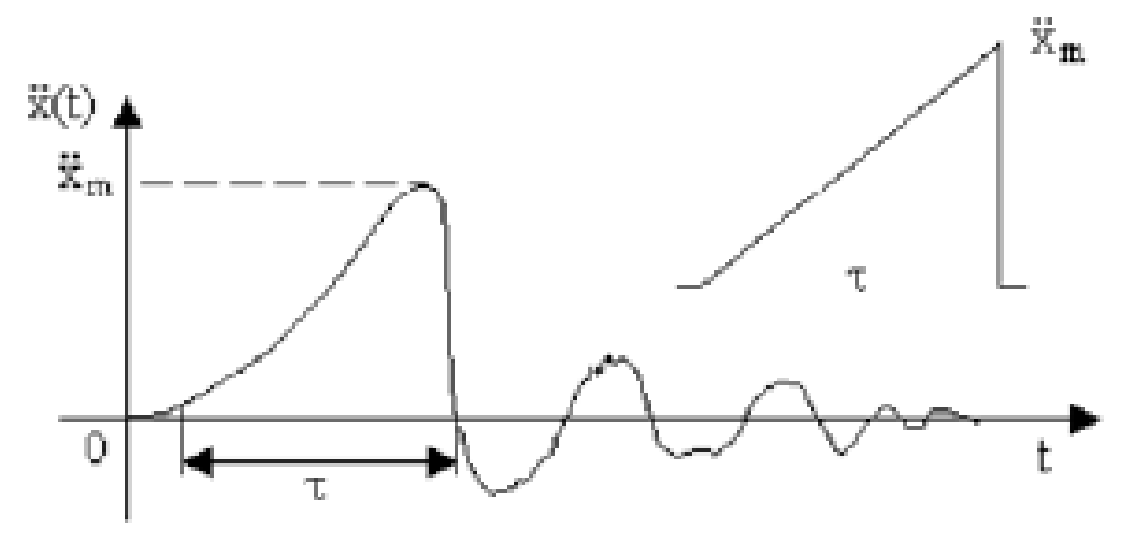

Figure 6 SHOCK RESistance SPECIFICATION TEST [51]

In robot servo actuators specification manuals and catalogues [52] there tends to be a specified vibration resistance and a shock resistance in $\mathrm{m} / \mathrm{s}^{2}$ as in Table 1 . These values are the environment conditions of the shaker machine under which the servo passed the shock test. Figure 6 shows an expected response to a simple shock produced by a shaker. Vibration resistance, on the other hand, are the shaker machine conditions used for the development of a Shock Response Spectrum (SRS) and under given conditions, the device is not likely to receive a response with a peak higher than created by the shaker machine. An example SRS is given in Figure 7 where the $\mathrm{x}$-axis represents the frequency of the input and the $\mathrm{y}$-axis are the magnitudes of the shock. 


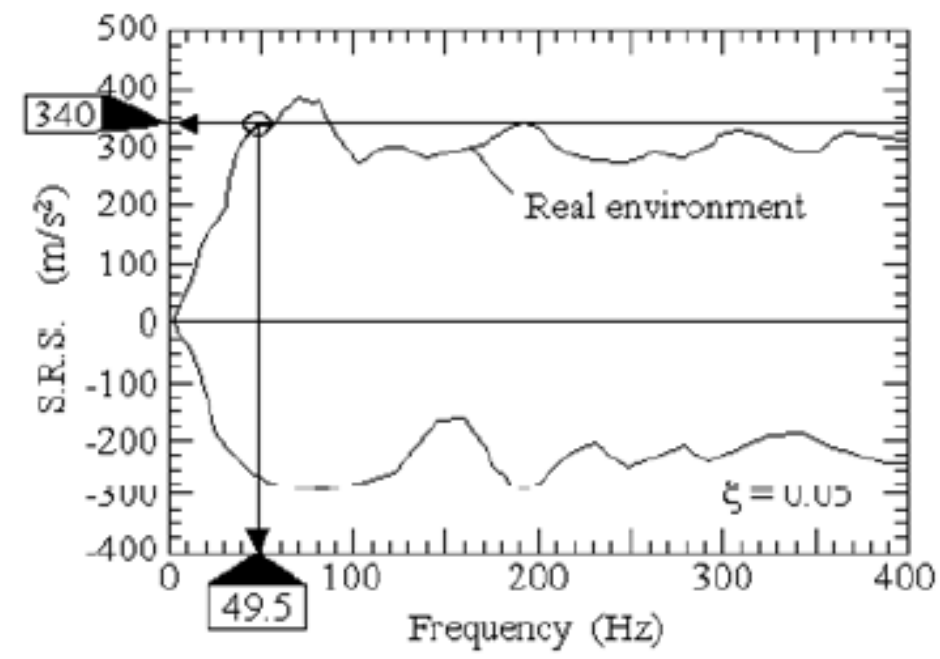

Figure 7 ENVIronMent SRS (POSITIVE AND Negative SPECTRA) [51]

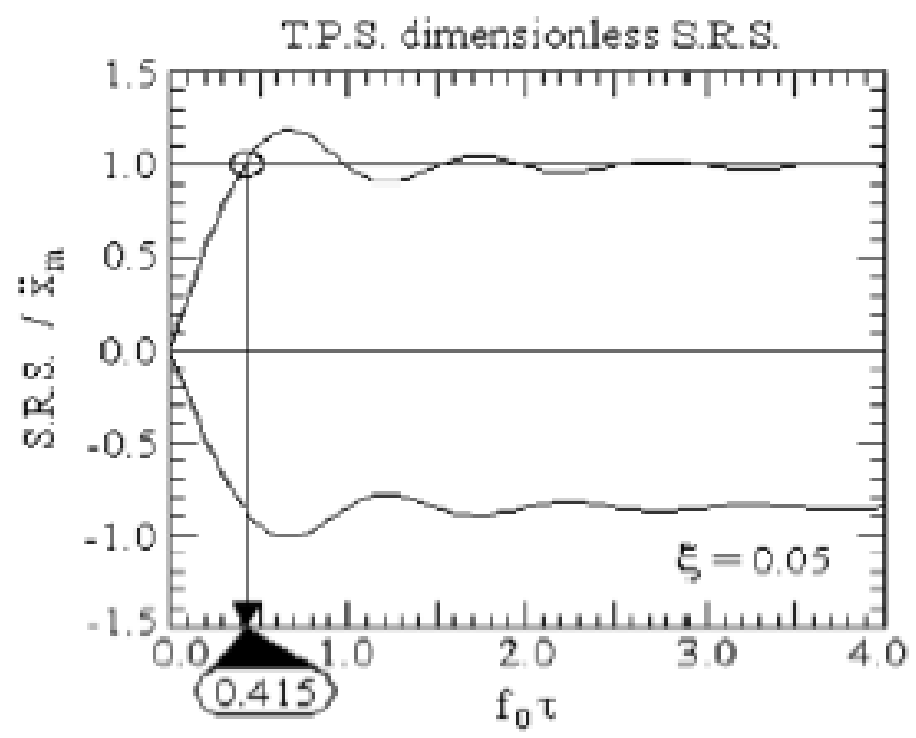

Figure 8 Dimensionless Terminal Peak SaWtooth SRS SCALED With THE AMPLITUDE AND FREQUENCY OF THE SRS [51] 
A dimensionless (normalized) version of the SRS in Figure 8 is also developed and then compared to simulated unit input models to determine a better approximation for the damping factor, $\zeta$, of the system and duration of the shock under various shock shapes. The frequencies corresponding to the first amplitude in Figure 8 having a unit amplitude for different shapes including the half-sine, TPS, and square shocks are compared against the reference shocks in Figure 9.

\begin{tabular}{|c|c|c|c|c|c|}
\cline { 3 - 6 } \multicolumn{2}{c|}{} & \multicolumn{4}{c|}{$\mathrm{f}_{01}$} \\
\hline $\mathrm{Q}$ & $\xi$ & Half-sine & Versed sine & TPS & Square \\
\hline 2 & 0.2500 & 0.413 & 0.542 & $/$ & 0.248 \\
\hline 3 & 0.1667 & 0.358 & 0.465 & 0.564 & 0.219 \\
\hline 4 & 0.1250 & 0.333 & 0.431 & 0.499 & 0.205 \\
\hline 5 & 0.1000 & 0.319 & 0.412 & 0.468 & 0.197 \\
\hline 6 & 0.0833 & 0.310 & 0.400 & 0.449 & 0.192 \\
\hline 7 & 0.0714 & 0.304 & 0.392 & 0.437 & 0.188 \\
\hline 8 & 0.0625 & 0.293 & 0.385 & 0.427 & 0.185 \\
\hline 9 & 0.0556 & 0.295 & 0.381 & 0.421 & 0.183 \\
\hline 10 & 0.0500 & 0.293 & 0.377 & 0.415 & 0.181 \\
\hline 15 & 0.0333 & 0.284 & 0.365 & 0.400 & 0.176 \\
\hline 20 & 0.0250 & 0.280 & 0.360 & 0.392 & 0.174 \\
\hline 25 & 0.0200 & 0.277 & 0.357 & 0.388 & 0.173 \\
\hline 30 & 0.0167 & 0.276 & 0.354 & 0.385 & 0.172 \\
\hline 35 & 0.0143 & 0.275 & 0.353 & 0.383 & 0.171 \\
\hline 40 & 0.0125 & 0.274 & 0.352 & 0.382 & 0.170 \\
\hline 45 & 0.0111 & 0.273 & 0.351 & 0.380 & 0.170 \\
\hline 50 & 0.0100 & 0.272 & 0.350 & 0.379 & 0.170 \\
\hline$\infty$ & 0.0000 & 0.267 & 0.344 & 0.371 & 0.167 \\
\hline
\end{tabular}

\section{Figure 9 VAlues of Dimensionless FreQuenCy CoRRESPONDING TO THE First}

\section{Passage OF The SRS by The Amplitude Unit [51]}

There are several technical reasons as to why shock testing is not popular for devices like the Harmonic Drive. Firstly, computer simulations of shocks have been shown to not resemble the real environment conditions accurately [51]. This means that experimental 
evaluations become necessary which is where the second issue comes into play - the fact that accurate measurements of changes in acceleration are still relatively difficult to gather experimentally.

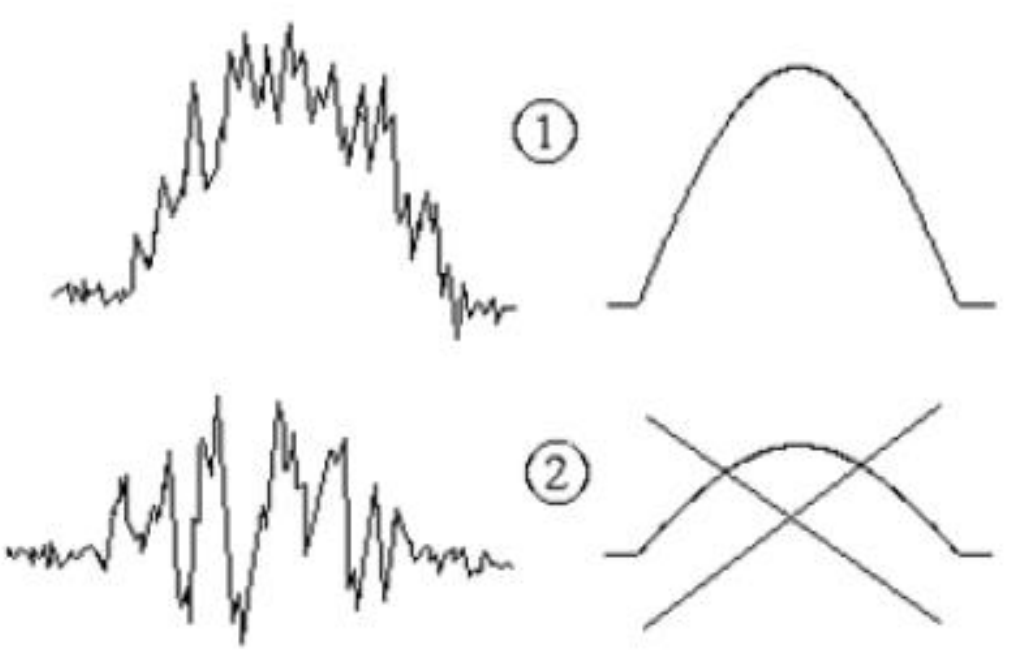

\section{Figure 10 Simple (1) ANd Complex (2) Shock Transformations USing The SAME}

\section{TrANSFORMATION METHOD [51]}

Lastly, a complex shock with many oscillations is much more common than a simple shock. Transforming complex shocks using the simple shock models and shapes is not necessarily valid and can lead to false assumptions about the device. Figure 10 compares the difficulties in both cases of a simple and complex shock. There are no empirical rules, however, on ensuring the quality of the shock in a simulated laboratory experiment [51], so the skill and judgement of the operator play a big role in ensuring integrity of the data.

Harmonic drives must be certified according to certain standards (e.g. MIL-STD, ISO9001, AS9100, etc.) depending on the area of application. While it is unclear why 
harmonic drive transmissions, specifically, are not tested using shaker machines, some quality assurance boards specify their overall goal when defining such standards which can provide a likely explanation. According to AS9100, the standard under which Harmonic Drive LLC is certified [53], the intended goal is for an organization to [54]

a) demonstrate its ability to consistently provide product that meets customer and applicable statutory and regulatory requirements, and

b) aims to enhance customer satisfaction through the effective application of the system, including processes for continual improvement of the system and the assurance of conformity to customer and applicable statutory and regulatory requirements.

From these statements, it is reasonable to assume that there was no technical justification found to conduct shock and vibration testing of harmonic drives by customers and users which likely includes reasons provided in the analysis of relevant literature provided in this chapter.

\subsection{Concluding Remarks}

Chapter 2 aims to introduce the reader to the very important and basic ideas of how impulse modelling and predictions are performed mathematically. The ideas of perfectly elastic and perfectly inelastic collisions are dispelled in this chapter to show that the rigid body assumption is not violated in such models and it remains consistent with the modelling of impulses performed later in this chapter. The rest of the chapter is an exploration into the field of shock theory and testing. There are many misconceptions and misnomers 
surrounding terms like collision, impact, shock, and impulse which have been defined and clarified to be used throughout the rest of this thesis. 


\section{MULTIPLE WORKING MODE APPROACH}

The development of the multiple working mode framework in a modular reconfigurable robot in [47] began due to an interest in having the robot perform tasks like door opening in uncontrolled environments. Any MRR module is able to switch online between active and passive modes as well as reconfigure to adapt to whatever task is being performed [47]. The

following section outlines the MRR module design, dynamics with impulse, and active/passive modes unification under the selection matrix.

\subsection{Module Design}

The schematic in Figure 11 shows the MRR module equipped with a brushless DC motor, harmonic drive, a torque sensor (with an amplifier), encoder, brake, and homing and limit sensors. The described hardware architecture can also be seen in Figure 12. 

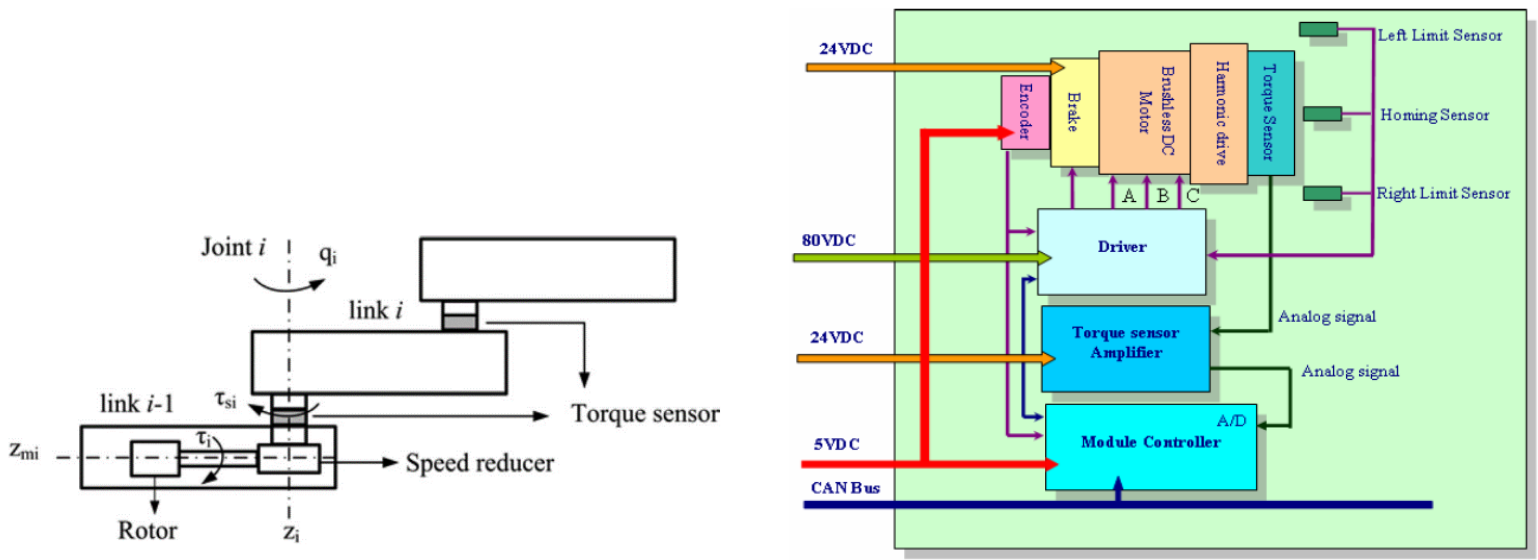

Figure 11 MRR Module SCHEMATIC [47]

Figure 12 HaRdWare Architecture [47]

The standard centralized control architecture cannot satisfy the self-containment requirement needed to create a modular unit, so some of the controls are distributed between the supervisory controller and a DSP-based module controller. To do this, the controls architecture is split into multiple layers as in Figure 13 which include the communication layer under the CAN Bus protocol and a decision layer that interprets commands from the supervisory controller. Next, the action layer performs tasks like homing, limiting, and calibration with a pre-set priority arbitrator determining what the user would have to do to activate such pre-programmed sub-routines. Driving electronics sit in the execution layer which uses PWM signal to drive the motor; the sensing layer consisting of a torque sensor, encoder, current sensor, homing and limit sensor provide feedback to both the module and supervisor controllers through the communication layer for on-line planning [47]. On-line trajectory planning and coordination is performed by centralized processing (supervisor) 
while module position control and friction compensation are performed by the module controller. Working modes for each module are also set by the supervisor.
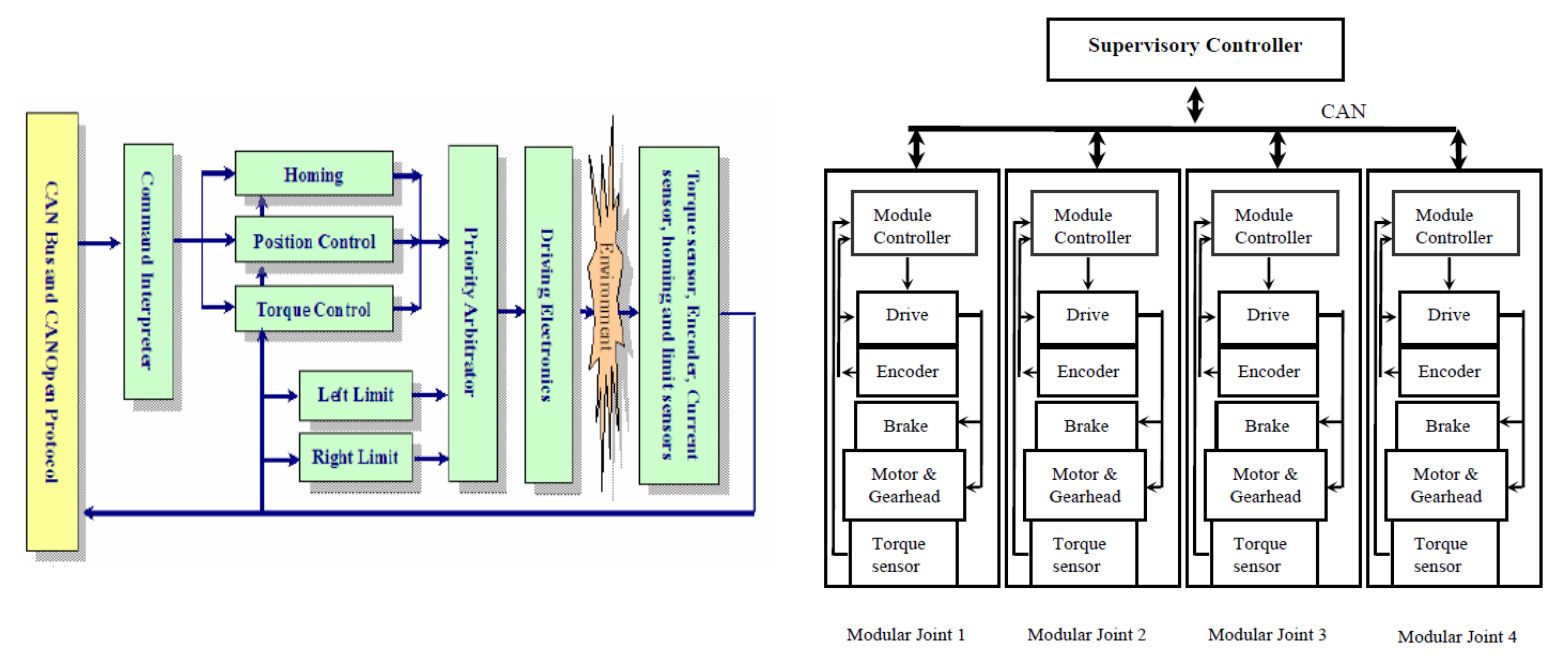

Figure 13 Control ARChitecture

LAYERING [47]

Figure 14 ConTrols ArChitecture [47]

What results is called a federated control architecture which can be seen in Figure 14 . Performing the hammering task with such an architecture can be done in several ways which are discussed in the following chapters after a thorough analysis of the impact dynamics.

The problem in implementing a passive mode in a joint is to compensate friction that prevents it from moving freely in the presence of an external force or a constraint. To do this, [47] proposes using a friction model based on the motion trend of the joint to estimate the amount of feedforward torque required to move the joint freely without additional mechanism or clutch systems. The joint can switch back and forth between active and passive modes seamlessly because the actuation chain is never interrupted. 
Friction modelling is an extensively studied topic [55], but because the friction compensation requirements for a joint working in passive mode is simply that the external force applied must be much larger than the friction, a relatively simple friction model can be used for the implementation of passive mode [47]. For a joint used in both high and low speed applications, the following model can be used to predict joint friction:

$$
\tau_{f}=\left[f_{c}+\left(f_{s}-f_{c}\right) e^{-f_{\tau} \dot{q}^{2}}\right] \operatorname{sgn}(\dot{q})+b \dot{q}
$$

Where $f_{c}, b, f_{s}$, and $f_{\tau}$ are the Coulomb, Viscous, Stribeck, and Static friction coefficients, respectively, $\dot{q}$ is the relative velocity of contact surfaces, and the sign function, $s g n$, is defined as

$$
\operatorname{sgn}(\dot{q})=\left\{\begin{array}{cc}
1 & \text { for } \dot{q}>0 \\
0 & \text { for } \dot{q}=0 \\
-1 & \text { for } \dot{q}<0
\end{array}\right.
$$

Equation (5) can be split into two components, a constant part and a variable part with the constant part usually dominating the total magnitude of the friction as seen in Figure 15. 


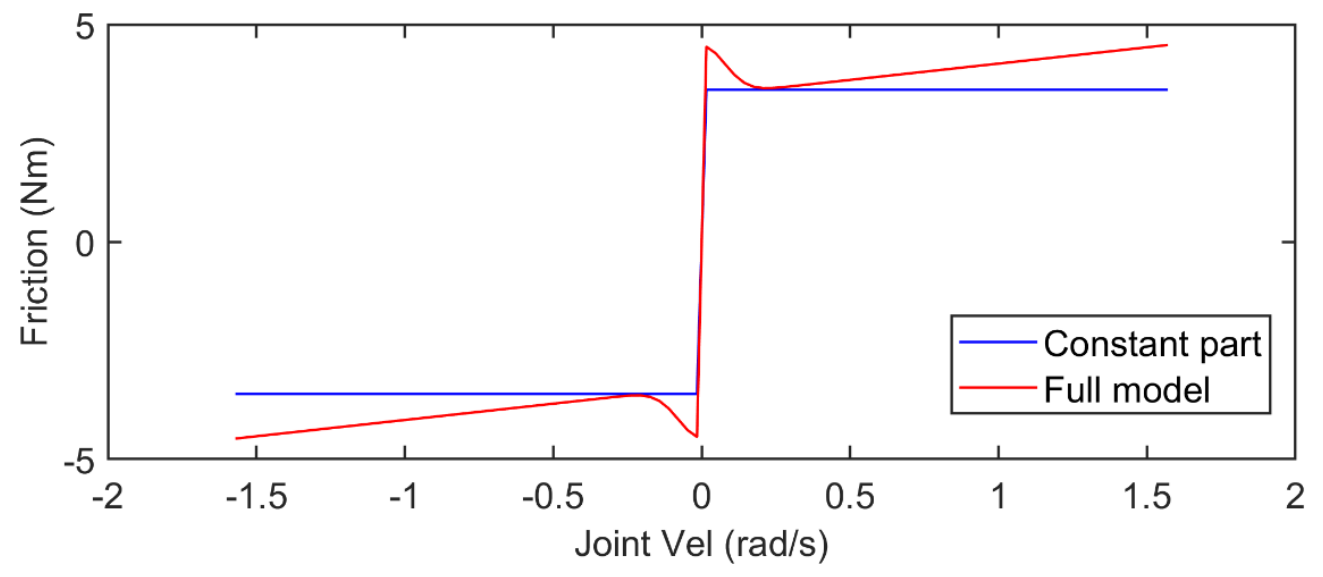

FigURE 15 Friction MODEL ILLUSTRATION [47]

If the motion trend for a task is assumed to be known or can be measured using a sensor like an encoder or torque sensor then a feedforward torque based on this trend can be applied to compensate the constant part:

$$
\check{\tau}_{f}=f_{m} \operatorname{sgn}(\dot{q})
$$

where $f_{m}$ is the coefficient of the constant part which is less than $f_{s}$, representing static friction. The approach was tested experimentally on a single base module based on the direction of rotation by pre-setting compensation currents in the motor to $-1.2 \mathrm{~A}$ for negative direction and $+1.5 \mathrm{~A}$ for positive direction. The joints were rotated with and without passive mode by applying an external torque, the results are tabulated below which show a substantial reduction in external torque needed to rotate the joint. 
TABle 2 EXTERnAl TORQUE FOR MRR MOdUle IN PASSIVE MODE [47]

\begin{tabular}{|c|c|c|}
\hline Rotate Direction & Positive $(\mathbf{0}$ to $360 \mathrm{deg})$ & Negative $(\mathbf{0}$ to $-\mathbf{3 6 0} \mathrm{deg})$ \\
\hline $\begin{array}{c}\text { Torque w/o compensation } \\
\text { (a) }\end{array}$ & $36.3 \mathrm{Nm}$ & $36.2 \mathrm{Nm}$ \\
\hline $\begin{array}{c}\text { Torque with compensation } \\
\text { (b) }\end{array}$ & $7.6 \mathrm{Nm}$ & $5.7 \mathrm{Nm}$ \\
\hline Ratio (b/a) & $21 \%$ & $16 \%$ \\
\hline
\end{tabular}

The addition of friction compensation into the passive mode means the joint is not fully, but partially passive. However, because the added input for the friction compensation is always less than the real joint friction, any energy added to the system is quickly dissipated. The aim of this friction compensation is only to reduce the effort required to move it, but not to eliminate that effort.

\subsection{Dynamics and Controls}

The Euler-Lagrange formulation of the robot dynamic equations with an external force applied at the end-effector are assumed to be given as

$$
M(q) \ddot{q}+h(\dot{q}, q)+\tau_{f}=\tau+J_{H}^{T} F_{e x t}
$$

where $M(q) \in \mathbb{R}^{n \times n}$ is the inertia matrix, $h(\dot{q}, q) \in \mathbb{R}^{n}$ is a vector containing gravity terms and non-inertial terms, $\tau_{f} \in \mathbb{R}^{n}$ is a vector of joint friction, $\tau \in \mathbb{R}^{n}$ is a vector of joint 
torques, $J_{H} \in \mathbb{R}^{m \times n}$ is the geometric Jacobian at the collision point, $F_{\text {ext }} \in \mathbb{R}^{m}$ is a force applied at the collision point, $q \in \mathbb{R}^{n}$ is the set of joint variables on the link-side, and $\dot{q}, \ddot{q}$ are its time derivatives.

Assuming joint torque estimates or measurements, $\tau_{s} \in \mathbb{R}^{n}$, are available either through joint torque sensors or other estimation methods, the link and motor dynamics can be separated and rewritten:

$$
\begin{gathered}
M_{l}\left(q_{l}\right) \ddot{q}_{l}+h_{l}\left(\dot{q}_{l}, q_{l}\right)-J_{H}^{T} F_{\text {ext }}=\tau_{s} \\
I_{m}\left(q_{m}\right) \ddot{q}_{m}+h_{m}\left(\dot{q}_{m}, q_{m}\right)+\tau_{f}+\tau_{s}=\Gamma \tau_{m}
\end{gathered}
$$

The subscript $l$ and $m$ represent link-side and motor-side dynamics terms, $I_{m} \in \mathbb{R}^{n \times n}$ is the motor inertia matrix and $h_{m} \in \mathbb{R}^{n}$ are motor non-linear and Coriolis terms vector as in [56], $\Gamma=\operatorname{diag}\left(\gamma_{1}, \ldots, \gamma_{n}\right) \in \mathbb{R}^{n \times n}$ is a diagonal matrix of motor gear ratios, and $\tau_{m} \in \mathbb{R}^{n}$ is a vector of motor input torques.

The formulation of the motor dynamics follows that of [56] and the joint modules are shown in Figure 11. The model includes the following three assumptions and set of notations:

A1. Each rotor is symmetric with respect to the axis of rotation.

A2. The torsion at each joint due to the flexibility of the torque sensor is small enough that it can be ignored, so the joint axis is regarded as a rigid one. 
A3. The transmitted force does not fail at the speed reducer, and the inertia between the torque sensor and the speed reducer is negligible.

$I_{m i} \quad$ Rotor moment of inertia about its axis of rotation

$\gamma_{i} \quad$ Reduction ratio of speed reducer

$q_{i} \quad$ Joint angle (link-side)

$\tau_{s i} \quad$ Coupling torque at torque sensor location

$\tau_{i} \quad$ Rotor output torque

$Z_{m i} \quad$ Unit vector along axis of rotation for the $i$ th rotor

$z_{i} \quad$ Unit vector along axis of rotation for the $i t h$ joint

For the first module, $i=1$

$$
I_{m 1} \gamma_{1}^{2} \ddot{q}_{m 1}+\gamma_{1} \tau_{f 1}+\tau_{s 1}=\gamma_{1} \tau_{m 1}
$$

For the second module, $i=2$

$$
I_{m 2} \gamma_{2}\left(\gamma_{2} \ddot{q}_{m 2}+z_{m 2}^{T} z_{1} \ddot{q}_{m 1}\right)+\gamma_{2} \tau_{f 2}+\tau_{s 2}=\gamma_{2} \tau_{m 2}
$$

For modules $i \geq 3$,

$$
I_{m i} \gamma_{i}\left(\gamma_{i} \ddot{q}_{m i}+\sum_{j=1}^{i-1} z_{m i}^{T} z_{j} \ddot{q}_{m j}+\sum_{j=2}^{i-1} \sum_{k=1}^{j-1} z_{m i}^{T}\left(z_{k} \times z_{j}\right) \dot{q}_{k} \dot{q}_{k}\right)+\gamma_{i} \tau_{f i}+\tau_{s i}=\gamma_{i} \tau_{m i}
$$

Equations (11) and (12) can be considered special cases of (13) where for $i=2$, the double summation term (Coriolis) gets excluded, and for $i=1$, both summation terms (Coriolis and 
inertial) are excluded. Note that for the remainder of the thesis, the subscript on $q_{l}$ is dropped in accordance with equation (8).

\subsubsection{Active Mode}

The Multiple Working Mode Approach consists of two working modes - active and passive. Active mode is used for position and torque control and is based on the distributed control scheme with torque sensing in [56]. Considering the system in (9) and (10), define the control law as

$$
\Gamma \tau_{m}=\tau_{m}^{a}=\hat{\tau}_{s}+\hat{\tau}_{f}+\hat{h}_{m}+u
$$

And the control input, $u$, as

$$
u=\hat{I}_{m} \ddot{q}_{d}-K_{D} \dot{e}-K_{P} e-u_{y}
$$

$\hat{\tau}_{s} \in \mathbb{R}^{n}$ is the joint torque estimate (from sensor or computational method), $\hat{\tau}_{f}$ represents friction models like in (5), $\hat{I}_{m}, \hat{h}_{m}$ are dynamic model parameters. The diagonal matrices $K_{D}, K_{P} \in \mathbb{R}^{n \times n}$ are positive definite control gains. The input $u_{y}$ is a parametric robust compensator as derived in [56] which compensates friction and manipulator configuration uncertainties; the derivation is omitted here. The following system errors are defined as. 


$$
\begin{gathered}
e=q-q_{d} \\
r=\dot{e}+\lambda e \\
a=\ddot{q}_{d}-2 \lambda \dot{e}-\lambda^{2} e
\end{gathered}
$$

where $e \in \mathbb{R}^{n}$ is the link-side joint position error, $q_{d} \in \mathbb{R}^{n}$ is the desired joint position, $r \in$ $\mathbb{R}^{n}$ and $a \in \mathbb{R}^{n}$ are mixed errors with $\dot{e} \in \mathbb{R}^{n}$ being the time derivative of $e$ and $\ddot{q}_{d} \in \mathbb{R}^{n}$ being the desired joint acceleration, while $\lambda$ is any positive constant.

The resulting dynamics reduce to

$$
\ddot{e}+I_{m}^{-1} K_{D} \dot{e}+I_{m}^{-1} K_{P} e=d
$$

The term $d \in \mathbb{R}^{n}$ represents the collection of uncompensated components due to unmodeled dynamics. Because the internal impulse on active joints is small, it is treated by the controller simply as part of $d$, otherwise, if internal impulses are predicted to be larger than a threshold value, then the joint is set into passive mode which is formulated next.

\subsubsection{Passive Mode}

The passive mode control law is defined as 


$$
\Gamma \tau_{\mathrm{m}}=\tau_{m}^{p}=\check{\tau}_{f}+\hat{h}_{m}
$$

The term $\check{\tau}_{f}$ is a static friction term so that $\left|\check{\tau}_{f}\right|<\left|\tau_{f}\right|$ according to (7). Setting $q_{d} \equiv q, \dot{q}_{d} \equiv$ $\dot{q}$, and $\ddot{q}_{d} \equiv 0$ results in $e \equiv 0$. The $\hat{\tau}_{s}$ term is excluded because the real joint torque is what actually drives the system and it is needed to be uncompensated.

Letting $\tilde{\tau}_{f}\left(\dot{e}_{s}\right)=\tau_{f}-\check{\tau}_{f}$ reduces the dynamics in (10) to

$$
I_{m} \ddot{q}_{m}+\tilde{\tau}_{f}\left(\dot{e}_{s}\right)+\tau_{s}=0
$$

The relation between equation (18) and external force at the end-effector is explained after the unification of the active and passive modes. Define $e_{s}=q-\frac{q_{m}}{\gamma}$ which leads to

$$
\ddot{e}_{s}+I_{m}^{-1} \tilde{\tau}_{f}\left(\dot{e}_{s}\right)+I_{m}^{-1} \tau_{s}=0
$$

where $e_{s} \in \mathbb{R}^{n}$ is the twist on the shaft on the link side. The last term, $I_{m}^{-1} \tau_{s}$, includes linkside inertia and dynamics forces which means the time constant for the passive mode is much larger than in (16), so the error $e_{s} \rightarrow 0$ as $t \rightarrow \infty$ very quickly.

\subsubsection{Selection Matrix}

A selection matrix, $S \in \mathbb{R}^{n \times n}$, is employed to switch each joint independently 


$$
S=\operatorname{diag}\left(s_{i}\right)
$$

where each column is a unit vector in $\mathbb{R}^{n}$ for a joint working in active mode. The final control law is thus

$$
\Gamma \tau_{m}=S \tau_{m}^{a}+(I-S) \tau_{m}^{p}
$$

Here, $I \in \mathbb{R}^{n \times n}$ is an identity matrix. To switch the $k$-th joint from active to passive mode is to flip the $k$-th column from a unit vector to a zero vector in the $S$-matrix. Substituting (14) and (25) into (10), the dynamics reduce to:

$$
S\left(\ddot{e}+I_{m}^{-1} K_{D} \dot{e}+I_{m}^{-1} K_{P} e\right)+(I-S)\left(\ddot{e}_{s}+I_{m}^{-1} \tilde{\tau}_{f}\left(\dot{e}_{s}\right)+I_{m}^{-1} \tau_{s}\right)=0
$$

This result is only possible if negligible error in the parameter estimates such that $d=$ 0 is assumed. The combination of the two controllers remain asymptotically stable, nevertheless, as per the specifications of both controllers.

\subsection{Concluding Remarks}

Chapter 3 presents how the multiple working mode approach is implemented into a modular reconfigurable robot. First, the overall hardware and controls architectures of the MRR are laid out to give an idea about how the joint is capable of performing in active and 
passive mode in a distributed manner. Next, the dynamics and controls of the robot are presented with a focus on the unification of the active and passive mode controls. 


\section{IMPULSE MODELS}

Chapter 3 laid the theoretical groundwork for the collision model of a simple particle. This chapter takes the expanded version of that theory as applied to a serial robot equipped with a hammer colliding with a nail.

\subsection{External Impulse Model}

When a robot end-effector collides with the environment as in Figure 16, it experiences an impulsive force (and torque) which is characterized by its short duration. If the robot and nail is assumed to be a system of rigid bodies, then the collision can be expressed using classical collision theory as in [33]:

$$
\left(\Delta v_{1}+\Delta v_{2}\right)^{T} \boldsymbol{n}=-(1+e)\left(v_{1}+v_{2}\right)^{T} \boldsymbol{n}
$$

Here, $\boldsymbol{n} \in \mathbb{R}^{m}$ is a vector perpendicular to the contact surface written with respect to the inertial frame, $v_{1}$ and $v_{2} \in \mathbb{R}^{m}$ are the pre-impact velocities of bodies 1 and 2 in task space, $\Delta v_{1}$ and $\Delta v_{2}$ represent the incremental changes in velocities of both bodies after impact, and $e$ is the coefficient of restitution. 


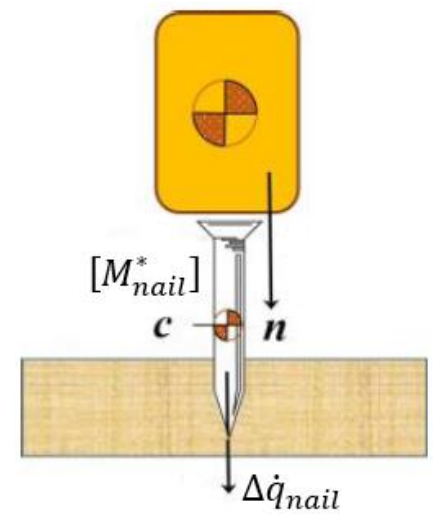

\section{Figure 16 HAMmer ANd NAIL ENVIRONMENT MODEL [39]}

Since the nail is static before the collision, $v_{2}=0$. The velocity of the end-effector, $v_{1}$, is selected by the user. The incremental change in the end-effector velocity can be found by considering the integral of (21) where $\tau_{s}$ is as defined in (9):

$$
\begin{gathered}
I_{m} \ddot{q}_{m}+\tilde{\tau}_{f}\left(\dot{e}_{s}\right)+M(q) \ddot{q}+h(\dot{q}, q)-J_{H}^{T} F_{\text {ext }}=0 \\
\int_{t}^{t+\Delta t}\left(\Gamma I_{m}+M\right) \ddot{q} d t+\int_{t}^{t+\Delta t} \tilde{\tau}_{f}\left(\dot{e}_{s}\right) d t+\int_{t}^{t+\Delta t} h(\dot{q}, q) d t \\
=\int_{t}^{t+\Delta t} J_{H}^{T} F_{\text {ext }} d t \\
\left(\Gamma I_{m}+M\right) \underbrace{[\dot{q}(t+\Delta t)-\dot{q}(t)]}_{\Delta \dot{q}}=J_{H}^{T} \hat{F}_{\text {ext }}
\end{gathered}
$$




$$
\begin{gathered}
\Delta \dot{q}=\left(\Gamma I_{m}+M\right)^{-1} J_{H}^{T} \widehat{F}_{e x t} \\
\Delta v_{1}=J_{H} \Delta \dot{q}=J_{H}\left(\Gamma I_{m}+M\right)^{-1} J_{H}^{T} \hat{F}_{e x t}
\end{gathered}
$$

The first term in (29) is obtained using the relationship $\ddot{q}_{m}=\Gamma \ddot{q}$. The integration occurs over a small period of time, $\Delta t$, during which all velocities remain finite, but it also means that the positions and orientations of all bodies have not changed much. Thus, integrals of any position-dependent terms like $h(\dot{q}, q)$ and $\tau_{f}$ are zero.

The incremental change of the velocity of the nail can be found using the effective mass model from [34]:

$$
\begin{gathered}
{\left[M_{\text {nail }}^{*}\right] \Delta \dot{q}_{\text {nail }}=\underbrace{F_{N} \cdot t_{s}}_{J_{\text {nail }}^{T}\left(-\hat{F}_{\text {ext }}\right)}} \\
\Delta v_{\text {nail }}=\frac{d_{i}}{t_{s}} \\
\Delta v_{2}=\Delta v_{\text {nail }}=J_{\text {nail }} \Delta \dot{q}_{\text {nail }}=J_{\text {nail }}\left[M_{\text {nail }}^{*}\right]^{-1} J_{\text {nail }}^{T}\left(-\hat{F}_{\text {ext }}\right)
\end{gathered}
$$

where $\left[M_{\text {nail }}^{*}\right] \in \mathbb{R}^{m \times n_{\text {nail }}}$ is the effective mass matrix, $\Delta q_{\text {nail }} \in \mathbb{R}^{n_{\text {nail }}}$ is the nail displacement variable, $F_{N} \in \mathbb{R}^{m}$ is the impulsive force acting on the nail head over the impact time, $t_{s}$, the term $\Delta v_{\text {nail }} \in \mathbb{R}^{m}$ is the nail velocity in task space, $d_{i} \in \mathbb{R}^{m}$ is the 
distance the nail travels, and $J_{\text {nail }} \in \mathbb{R}^{m \times n_{\text {nail }}}$ is the nail Jacobian. Here, the nail is modelled as a prismatic joint with $n_{\text {nail }}=1$ where it can slide along the $\mathrm{z}$-direction of the normal vector, $\boldsymbol{n}$. If the impulsive force, $F_{N}$, can be measured using a force sensor, then it can be used with (34) to calculate the effective the effective mass matrix, [ $\left.M_{\text {nail }}^{*}\right]$.

Substituting (32) and (35) into (27) and then solving for the impulse leaves:

$$
\hat{F}_{\text {ext }}=\left\{\frac{-(1+e)\left(v_{1}\right)^{T} \boldsymbol{n}}{\boldsymbol{n}^{T}\left\{J_{H}\left(\Gamma I_{m}+M\right)^{-1} J_{H}^{T}+J_{\text {nail }}\left[M_{\text {nail }}^{*}\right] J_{\text {nail }}^{T}\right\} \boldsymbol{n}}\right\} \boldsymbol{n}
$$

The term inside the big bracket is the magnitude of the impulse. Note that the vector, $\widehat{F}_{\text {ext }}$, is an $m$-vector just like $F_{\text {ext }}$ which includes both the impulse forces and torques at the end-effector, written w.r.t the base frame.

\subsection{Internal Impulse Model}

When the end-effector experiences an external impulsive force, the joints must react to keep the joint motion constrained. These reaction loads are called internal impulses. In order to calculate the internal impulses on each joint as a function of the external impulse, the internal reaction impulses have to be exposed. The Newton-Euler formulation from [57] is adopted here with a small change about the joint constraint. In the original formulation it is assumed that a revolute joint produces no impulsive torque about the $\mathrm{z}$-axis and is free to rotate, but it is constrained here to estimate the impulsive torque in the speed reducer. 
Consider the $i$-th system of modules like in Figure 11 with impulsive and non-impulsive forces and torques applied at the joint center as in Figure 17.

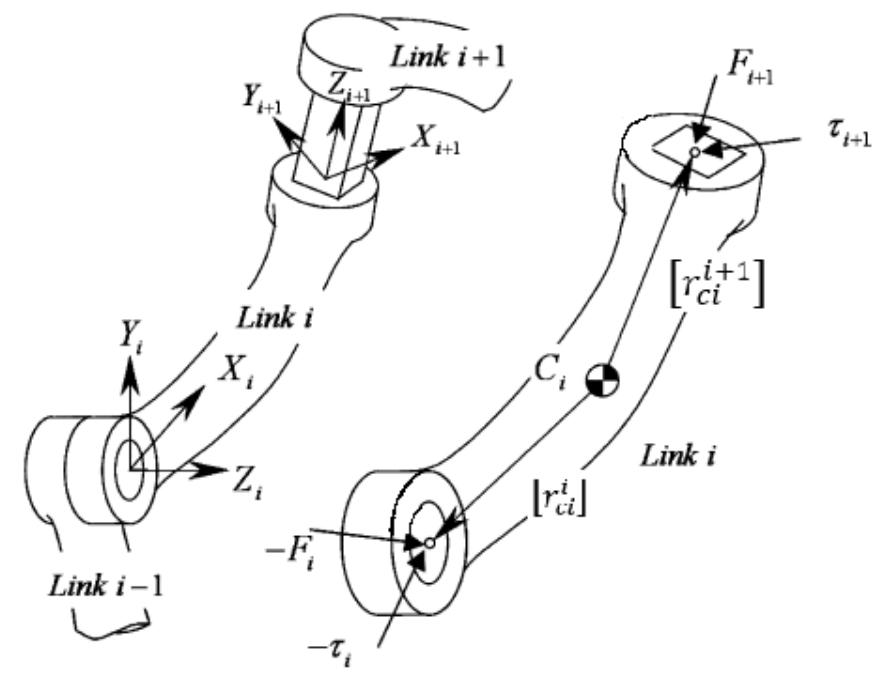

Figure 17 Force/Torque Model of a Serial Chain Robot [57]

The following derivation can be applied to both 2D and 3D space, each further subdivided into $\mathbb{R}^{m_{v}}$ and $\mathbb{R}^{m_{\omega}}$. In $2 \mathrm{D}$ space, $m_{v}=2$ and $m_{\omega}=1$ since there are two velocity components in the $\mathrm{x}-\mathrm{y}$ place and one angular velocity about the z-axis. In 3D space, both are equal to three.

$$
\begin{gathered}
m_{i} \dot{v}_{c i}=-F_{i}+R_{i+1}^{i} F_{i+1}^{i+1}+f_{i}^{\Sigma} \\
I_{c i} \dot{\omega}_{i}+\omega_{i} \times\left[I_{c i} \omega_{i}\right]=-\tau_{i}+R_{i+1}^{i} \tau_{i+1}^{i+1}-r_{c i}^{i} \times F_{i}+r_{c i}^{i+1} \times\left[R_{i+1}^{i} F_{i+1}^{i+1}\right]+\tau_{i}^{\Sigma}
\end{gathered}
$$


Newton-Euler equations are formulated in frame $i$, so for the rest of this derivation, unless otherwise specified, the dynamics terms are written with a subscript $i$ and no superscript to mean w.r.t. frame $i$.

In (37) and (38), $m_{i}$ and $I_{c i} \in \mathbb{R}^{m_{\omega}}$ are scalar link mass and inertia matrix about the center of gravity, $v_{c i} \in \mathbb{R}^{m_{v}}$ and $\omega_{i} \in \mathbb{R}^{m_{\omega}}$ are the velocity and angular velocity of the link center of gravity, $F_{i}, F_{i+1}^{i+1} \in \mathbb{R}^{m_{v}}$ and $\tau_{i}, \tau_{i+1}^{i+1} \in \mathbb{R}^{m_{\omega}}$ are the impulsive forces and torques applied at the center of joint $i$ and $i+1$, respectively, $R_{i+1}^{i} \in \mathbb{R}^{m_{v}}$ is the rotation matrix transforming the $(i+1)$ th coordinate system to the $i$ th, and $f_{i}^{\Sigma} \in \mathbb{R}^{m_{v}}$ and $\tau_{i}^{\Sigma} \in \mathbb{R}^{m_{\omega}}$ are the sum of all the non-impulsive forces and torques exerted at the joints. $r_{c i}^{i} \in \mathbb{R}^{m_{v}}$ is the vector point from the center of gravity to the center of joint $i$, and $r_{c i}^{i+1}$ towards joint $i+1$.

By integrating (37) and (38) with respect to time, the incremental changes in velocity and angular velocity of the link center of gravity can be obtained:

$$
\begin{gathered}
m_{i} \Delta v_{c i}=-\hat{F}_{i}+R_{i+1}^{i} \hat{F}_{i+1}^{i+1} \\
I_{c i} \Delta \omega_{i}=-\hat{\tau}_{i}+R_{i+1}^{i} \hat{\tau}_{i+1}^{i+1}-\left[r_{c i}^{i}\right] \hat{F}_{i}+\left[r_{c i}^{i+1}\right]\left[R_{i+1}^{i} \hat{F}_{i+1}^{i+1}\right]
\end{gathered}
$$

The matrices $\left[r_{c i}^{i}\right]$ and $\left[r_{c i}^{i+1}\right]$ are skew symmetric matrices corresponding to cross products $r_{c i}^{i} \times$ and $r_{c i}^{i+1} \times$ 
Next, the incremental velocity and angular velocity changes are related to the vector of independent joint velocities, $\dot{q}$. To do this, a Jacobian matrix for the center of mass of each link, $J_{c i}=\left[\begin{array}{c}J_{c i}^{v} \\ J_{c i}^{\omega}\end{array}\right] \in \mathbb{R}^{m \times n}$, is defined and then the expression for velocity of the center of gravity with respect to the base frame is written as follows:

$$
\begin{gathered}
\Delta v_{c i}^{0}=R_{i}^{0} \Delta v_{c i}^{i}=J_{c i}^{v} \Delta \dot{q} \\
\Delta \omega_{i}^{0}=R_{i}^{0} \Delta \omega_{i}^{i}=J_{c i}^{\omega} \Delta \dot{q} \\
\Delta v_{c i}^{i}=\left[R_{i}^{0}\right]^{T} J_{c i}^{v} \Delta \dot{q} \\
\Delta \omega_{i}^{i}=\left[R_{i}^{0}\right]^{T} J_{c i}^{\omega} \Delta \dot{q}
\end{gathered}
$$

From (31), the relation between the incremental changes in joint velocities and the external impulse is already known, therefore

$$
\begin{aligned}
& \Delta v_{c i}^{i}=\left[R_{i}^{0}\right]^{T} J_{c i}^{v}\left(\Gamma I_{m}+M\right)^{-1} J_{H}^{T}\left(\begin{array}{l}
\hat{F} \\
\hat{\tau}
\end{array}\right)_{\text {ext }} \\
& \Delta \omega_{i}^{i}=\left[R_{i}^{0}\right]^{T} J_{c i}^{\omega}\left(\Gamma I_{m}+M\right)^{-1} J_{H}^{T}\left(\begin{array}{l}
\hat{F} \\
\hat{\tau}
\end{array}\right)_{\text {ext }}
\end{aligned}
$$

Here, the vector $\widehat{F}_{\text {ext }}$ is expanded into its force and torque components to match the NewtonEuler equations. 
The left-hand side of (39) and (40) can be simplified as follows:

$$
\begin{aligned}
& m_{i} \Delta v_{c i}^{i}=m_{i}\left[R_{i}^{0}\right]^{T} J_{c i}^{v}\left(\Gamma I_{m}+M\right)^{-1} J_{H}^{T}\left(\begin{array}{l}
\hat{F} \\
\hat{\tau}
\end{array}\right)_{\text {ext }}=\left[V_{i}\right]\left(\begin{array}{l}
\hat{F} \\
\hat{\tau}
\end{array}\right)_{\text {ext }} \\
& I_{c i} \Delta \omega_{i}=I_{c i}\left[R_{i}^{0}\right]^{T} J_{c i}^{\omega}\left(\Gamma I_{m}+M\right)^{-1} J_{H}^{T}\left(\begin{array}{l}
\hat{F} \\
\hat{\tau}
\end{array}\right)_{\text {ext }}=\left[W_{i}\right]\left(\begin{array}{l}
\hat{F} \\
\hat{\tau}
\end{array}\right)_{\text {ext }}
\end{aligned}
$$

The matrices $\left[V_{i}\right] \in \mathbb{R}^{\left(n \cdot m_{v}\right) \times m}$ and $\left[W_{i}\right] \in \mathbb{R}^{\left(n \cdot m_{\omega}\right) \times m}$ are unitless and configuration dependent.

Now, the $n$th link experiences no impulses $\hat{F}_{n+1}$ or $\hat{\tau}_{n+1}$ so these will be zero vectors. However, the end-effector does experience external impulses $\hat{F}_{\text {ext }}$ and $\hat{\tau}_{\text {ext }}$, so the equations for the $n$th link can be written as:

$$
\begin{gathered}
{\left[V_{n}\right]\left(\begin{array}{l}
\hat{F} \\
\hat{\tau}
\end{array}\right)_{\text {ext }}=-\hat{F}_{n}+R_{0}^{n} \hat{F}_{\text {ext }}} \\
{\left[W_{n}\right]\left(\begin{array}{l}
\hat{F} \\
\hat{\tau}
\end{array}\right)_{\text {ext }}=-\hat{\tau}_{n}+R_{0}^{n} \hat{\tau}_{\text {ext }}-\left[r_{c n}^{n}\right] \hat{F}_{n}+\left[r_{c n}^{n+1}\right]\left[R_{0}^{n} \hat{F}_{\text {ext }}\right]}
\end{gathered}
$$

Finally, equations (39), (40), (46) and (47) can be written in matrix form:

$$
[D]\left(\begin{array}{c}
\hat{F} \\
\hat{\tau}
\end{array}\right)_{\text {ext }}=[A]\left(\begin{array}{c}
\hat{F} \\
\hat{\tau}
\end{array}\right)+[B]\left(\begin{array}{c}
\hat{F} \\
\hat{\tau}
\end{array}\right)_{\text {ext }}
$$


Where $\quad$ the $\quad$ vectors $\quad \hat{F}=\left(\left(\hat{F}_{1 x}, \hat{F}_{1 y}, \hat{F}_{1 z}\right)^{T}, \ldots, \hat{F}_{n}^{T}\right)^{T} \in \mathbb{R}^{n \cdot m_{v}} \quad$ and $\quad \hat{\tau}=$ $\left(\left(\hat{\tau}_{1 x}, \hat{\tau}_{1 y}, \hat{\tau}_{1 z}\right)^{T}, \ldots, \hat{\tau}_{n}^{T}\right)^{T} \in \mathbb{R}^{n \cdot m_{\omega}}$ are the internal joint impulses. The sub-matrix $[D] \in$ $\mathbb{R}^{(m \cdot n) \times m}$ is a block matrix including $\left[V_{i}\right]$ and $\left[W_{i}\right]$ matrices, and the matrix $[B] \in \mathbb{R}^{(m \cdot n) \times m}$ only has the entries in the $n$th and $(n+n)$ th rows relating to the external impulse at the endeffector in (46), (47):

$$
[D]=\left[\begin{array}{c}
V_{1} \\
\vdots \\
V_{n} \\
W_{1} \\
\vdots \\
W_{n}
\end{array}\right],[B]=\left[\begin{array}{cc}
0 & 0 \\
\vdots & \vdots \\
R_{0}^{n} & 0 \\
0 & 0 \\
\vdots & \vdots \\
{\left[r_{c n}^{n+1}\right]\left[R_{0}^{n}\right]} & R_{0}^{n}
\end{array}\right]
$$

The matrix $[A] \in \mathbb{R}^{(m \cdot n) \times(m \cdot n)}$ consists of three parts

$$
A=\left[\begin{array}{cc}
{\left[A_{F F}\right]} & {[0]} \\
{\left[A_{T F}\right]} & {\left[A_{T T}\right]}
\end{array}\right]
$$

where the sub-matrices are defined as

$$
A_{F F}=A_{T T}=\left[\begin{array}{cccccc}
-\boldsymbol{I}_{3 \times 3} & R_{2}^{1} & 0 & 0 & 0 & 0 \\
0 & -\boldsymbol{I}_{3 \times 3} & \ddots & 0 & 0 & 0 \\
0 & 0 & \ddots & R_{i+1}^{i} & 0 & 0 \\
0 & 0 & 0 & -\boldsymbol{I}_{3 \times 3} & \ddots & 0 \\
0 & 0 & 0 & 0 & \ddots & R_{n}^{n-1} \\
0 & 0 & 0 & 0 & 0 & -\boldsymbol{I}_{3 \times 3}
\end{array}\right]
$$




$$
A_{T F}=\left[\begin{array}{cccccc}
{\left[r_{c 1}^{1}\right]} & {\left[r_{c 1}^{2}\right] R_{2}^{1}} & 0 & 0 & 0 & 0 \\
0 & {\left[r_{c 2}^{2}\right]} & \ddots & 0 & 0 & 0 \\
0 & 0 & \ddots & {\left[r_{c i}^{i+1}\right] R_{i+1}^{i}} & 0 & 0 \\
0 & 0 & 0 & {\left[r_{c i}^{i}\right]} & \ddots & 0 \\
0 & 0 & 0 & 0 & \ddots & {\left[r_{c(n-1)}^{n}\right] R_{n}^{n-1}} \\
0 & 0 & 0 & 0 & 0 & {\left[r_{c n}^{n}\right]}
\end{array}\right]
$$

The vector of internal impulses on the joints can thus be calculated using

$$
\left(\begin{array}{l}
\hat{F} \\
\hat{\tau}
\end{array}\right)=\underbrace{[A]^{-1}([D]-[B])}_{[L]}\left(\begin{array}{l}
\hat{F} \\
\hat{\tau}
\end{array}\right)_{\text {ext }}
$$

where $[L] \in \mathbb{R}^{(m \cdot n) \times m}$ is the mapping between internal joint impulse and external impulse on the hammer. This model provides a solution for the magnitudes of internal impulse forces and torques for any $n$-link serial manipulator with rigid links and joints in $2 \mathrm{D}$ or $3 \mathrm{D}$ space.

\subsection{Concluding Remarks}

Chapter 4 lays out the detailed analysis of an MRR collision with an external mass. The theory from Chapter 2 is utilized to create analytical models for external and internal impulses of the robot. External impulse model generates solutions for the impulse at the tool with the support of the effective mass model which accounts for environmental properties like hardness. Internal impulse model combined with the analysis of harmonic drive structure in Chapter 2 can provide an effective way to determine the acceptable joint velocities and configurations in which the joint does not outright break during collision. 


\section{HAMMERING STRATEGY}

Performing one heavy swing that drives the nail all the way into a board may be dangerous depending on the board material hardness. Instead, it is assumed that multiple swings are performed until the nail is driven all the way into the board. Consider the sequence in Figure 18 where the hammer is swung over again until the nail head is only a small distance above the board. If the hammer were to be swung a fourth time, it would encounter the board. The board may be assumed to have a finite or an infinite effective mass. If the board material is soft like Styrofoam or balsa wood, then it's better to consider the surface to have some tested finite mass, otherwise the material will likely get damaged. In the case of hardwood or concrete, however, damage to the surface is unlikely, so the more conservative assumption of infinite mass is reasonable.

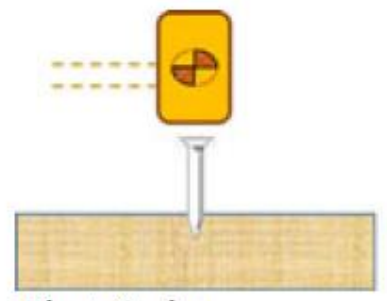

First Swing.

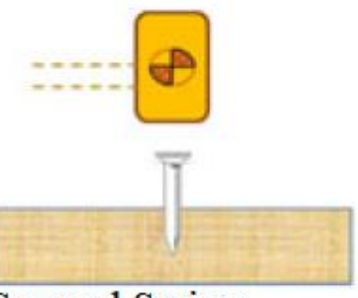

Second Swing
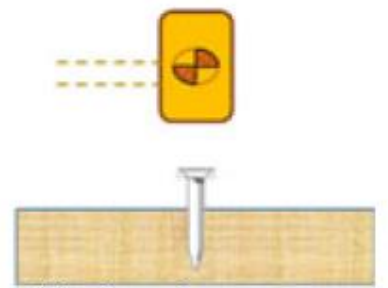

Third Swing

\section{Figure 18 HAMMERING TASK [39]}

According to (36), if the end-effector collides with the board, the second term inside the denominator relating to the environment will be reduced to zero. This results in higher end-effector external impulse regardless of the configuration or velocity of the joints: 


$$
\widehat{F}_{e x t}=\left\{\frac{-(1+e)\left(v_{1}\right)^{T} \boldsymbol{n}}{\boldsymbol{n}^{T}\left\{J_{H}\left(\Gamma I_{m}+M\right)^{-1} J_{H}^{T}\right\} \boldsymbol{n}}\right\} \boldsymbol{n}
$$

The same can be reached by setting $\Delta v_{2}=0$ since the wall does not have changes in velocity.

Optimal configuration at the time of collision is a well-studied topic. [32] [34] [58] have proposed a number of measures like the dynamic impact ellipsoid, normalized impact geometry, and the internal impact measure. The internal impact measure from [34] is based off the relationship in (53):

$$
w_{i i}=\sqrt{\operatorname{det}\left(L^{T} L\right)}=\sigma_{1} \sigma_{2} \ldots \sigma_{n}
$$

where $w_{i i}$ is a product of $\sigma_{1} \sigma_{2} \ldots \sigma_{n}$ that represent magnitudes of axes of the dynamic impact ellipsoid. The measure in (55) represents the relative magnitudes of internal impulses corresponding to a unit change in external impulse.

Each swing is separated into three common phases: pre-impact, impact, and postimpact. In the pre-impact phase, the robot generates trajectories in active mode based on the internal impact measure (55), external impulse (36), and internal impulse (53). Moments before impact, the joints with large expected internal impulses are set into passive mode by their respective controllers. In the impact and post-impact phases, the passive joints simply 
experience an impulsive input load which must be below the allowable specifications of the transmission.

In the case of a harmonic drive transmission, the specifications are provided by the manufacturer [50] as a limit on the Momentary Peak Torque, $T_{M P T}$, duration of impact, $t_{s}$, and the motor rpm, $n_{s}$. The limits are imposed on the system based on the provided equations:

$$
N_{S} \leq \frac{10^{4}}{2\left(\frac{n_{s} \gamma}{60}\right) t_{s}} \leq 1 \times 10^{4}
$$

And

$$
T_{S} \leq T_{M P T}
$$

$N_{S}$ represented the maximum number of impact occurrences for an infinite fatigue life of the harmonic drive, $n_{S}$ is the motor rpm, $\gamma$ is the gear ratio, and $T_{S}$ is the reaction (load) torque of the harmonic drive at impact.

The momentary peak torque limit should not be exceeded except in rare, emergency situations, so the number of occurrences the harmonic drive experiences it must be minimized. Instead, the Repeated Peak Torque value, $T_{R P T}$, is selected as the limit for each swing which is a safe limit with infinite fatigue life of the transmission as seen in Figure 19. 
Duration of impact, $t_{s}$, may be determined experimentally or by using estimates from the specifications manual. In [35], each impact lasted approximately $50 \mathrm{~ms}$ according to a piezoresistive F/T sensor data (model FLX-201-D). The specification manual provided an example with a $150 \mathrm{~ms}$ impact duration [50]. Shorter impact duration is a more conservative choice.

Equation (57) is essentially the reaction torque of the harmonic drive. If it were measured over the impact duration, it would have a similar shape as the external impulsive torque. To be conservative, it is assumed that the external impulsive torque is a square waveform. Thus, the maximum reaction impulse, $\hat{\tau}_{n z_{\text {max }}}$, that the harmonic drive can exert before damage is the integral of the reaction torque over time:

$$
\hat{\tau}_{n z_{\max }}=\int T_{R P T} d t=T_{R P T} \cdot t_{s}
$$

For a square waveform, the area under the curve is simply the product of the maximum reaction torque and impact duration. Alternatively, a sinusoidal shape of external impulsive force may also be assumed.

Now, equation (56) is rearranged for the maximum input rpm, $n_{s}$, at impact. Then, pre-multiply both sides by $2 \pi / 60$ to convert it into radians per second. Lastly, divide by the gear ratio, $\gamma$, to obtain the rotational speed of the output when impact occurs. 


$$
\left|\dot{q}_{i}\right|=\left(\frac{\pi}{t_{s} \gamma}\right)
$$

Equation (59) is the magnitude of link velocity at impact for the $i$ th joint. An $n$-joint with all joints moving at this maximum speed will have end-effector velocity of:

$$
\left|v_{1}\right|=J_{H}|\dot{q}|=J_{H}\left(\frac{\pi}{t_{S}}\right) \Gamma^{-1}
$$

Lastly, the joint velocities defined in (59) must have a direction or can also be set to zero by multiplying the equation by 1,0 , or -1 .

The maximum internal impulse in (58) functions as an upper limit for the system that may not be exceeded under any circumstance. If this value is exceeded, the end-effector velocity must be reduced until the values are below the RPT threshold. While below the RPT limit, but above the Rated Torque limit, $\hat{\tau}_{n z}$, passive mode is selected to reduce torque build-up. While below the limit defined by the Rated Torque, the joint may be left in active mode as illustrated in Figure 19. Note that the velocity is limit in (60) is a sort of lower limit determined by how many impact occurrences above the RPT threshold are expected. Setting $N_{S}=1 \times 10^{4}$ implies that many such occurrences are expected, making this a very conservative assumption. Reducing $N_{S}$ will result in higher link velocities at impact but increasing $N_{S}$ any higher will make the motion of the end-effector so slow that the collision 
would look more like a soft touch to the surface and the robot would come to a static equilibrium with the environment.

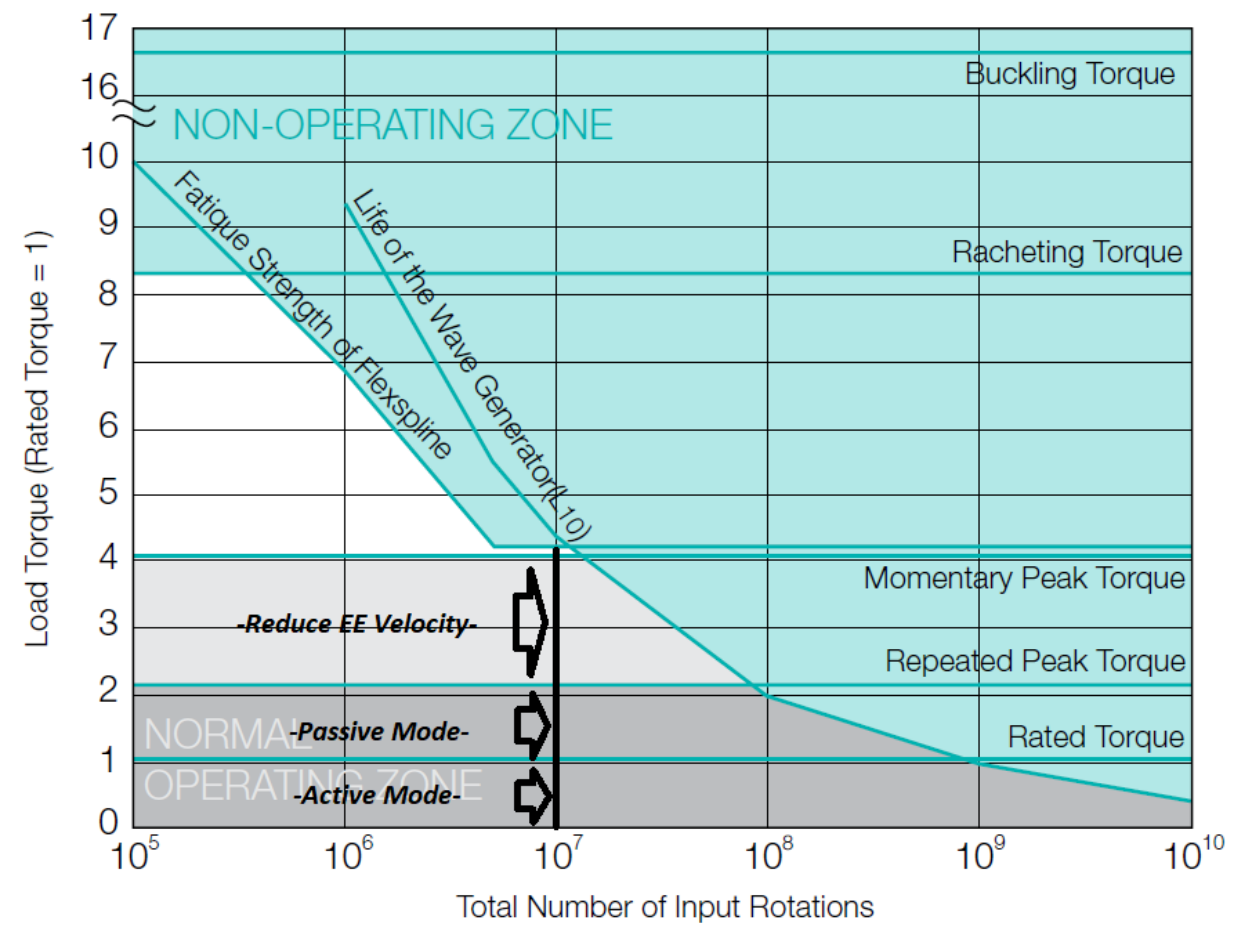

Figure 19 Harmonic Drive Limit Torques ANd Mode SWITCHING

To determine when the robot should switch joints into passive mode, two items are tracked: links momentum and end-effector pose:

$$
\begin{gathered}
P=M(q) \dot{q} \geq M\left(q_{f}\right) \dot{q}_{\max } \\
\left|x_{e}^{0} \cdot x_{n}^{0}\right| \leq\left|x_{s}^{0} \cdot x_{n}^{0}\right| \leq 1
\end{gathered}
$$


Tracking only the momentum, $P$, can lead to false positives because there may be combinations of configurations and joint velocities throughout the trajectory that produce the final values at impact. In (62), $x_{e}^{0}$ and $x_{n}^{0}$ are the nail and end-effector pose in the inertial frame and (62) is their projection on each other. $x_{s}^{0}$ is the pose of the surface of the hammer which meets the nail before the end-effector frame which serves as a lower limit for (62). Using the projection is better than tracking error $e_{r}=\left(x_{e}^{0}-x_{n}^{0}\right)$ because not only the direction but also the magnitude of every entry must be checked which isn't as reliable and quick computationally. When both conditions are satisfied, joints that exceed (58) are set into passive mode.

The swinging strategy may be summarized with the following steps:

1) Find the configuration which maximizes (55) for a given end-effector constraint in task-space (nail location), $x_{e}=f\left(q_{f}\right)$.

2) Choose the end-effector velocity as its maximum value defined by (60) then estimate the external impulse on the end-effector and internal impulse on the joints in (36) and (53), respectively.

3) If the entries in (53) corresponding to joint torques are below $\hat{\tau}_{n z_{\max }}$, but above $\hat{\tau}_{n z_{R T}}$, store their indices in an array and generate smooth polynomial trajectories. Otherwise, reduce the end-effector velocity until the condition is satisfied. Joints that do not exceed the rated torque limit are left in active mode.

4) Let the joint controllers switch their respective joints into passive mode by changing the selection matrix when (61) and (62) are satisfied. 
For a non-redundant manipulator in 3D space, step 1) is completed by solving the inverse kinematics problem, $q_{f}=f^{-1}\left(x_{e}\right)$, and selecting the solution for which the internal impact measure, $w_{i i}$, is the largest. Then, assume the coefficient of restitution, $e$, is its maximum value of 1 (can be adjusted based on testing) and the velocity of the end-effector at the

moment of impact is chosen as $v_{e}=J_{H}\left(q_{f}\right) \dot{q}_{\max }$. The external and internal impulse forces/torques are then estimated using their respective equations and the indices of joints with high impulses about the joint axis are stored in a separate array. If the estimated internal impulses are within the threshold, the trajectory is executed and the joints in the stored array are switched into passive mode.

\subsection{Hammering Experiment}

To study the efficacy of impulse models and hammering strategy proposed in this thesis, an experimental study of an MRR using the multiple working mode framework is conducted.

An external $\mathrm{F} / \mathrm{T}$ sensor is used to collect data throughout the collision to determine modelling accuracy and precision. Because the sensor is only able to collect impulsive force and torque data, that information must be integrated over the impact duration and then compared to the model estimate. Due to hardware limitations, only the external impulse model and the effective mass model can be tested in this manner. Joint torque sensor data would be needed to evaluate internal impulse on the joints. Instead, encoder measurements of joint velocities are compared against predicted changes in joint velocity for joint 2 and 3 
using equation (31). This also allows the evaluation of the effectiveness of the passive working mode. By setting joint 2 into active mode and joint 3 into passive mode, the differences in response of each module can be exposed. The effectiveness of passive mode is evaluated based on potential stress on the harmonic drive bearing and flexspline during the collision that are not due to joint reactions, but due to the motor input. The unavailability of joint torque measurements also means that joint 3 is running in passive mode with no friction compensation. This is not a problem for the experiment or the multiple working mode framework because one, it is assumed that the collision is so short that the motor cannot act quickly enough to compensate, and two, the on-board DSP still allows the joint to rotate, just with more friction.

The rest of the chapter presents the hardware parameters of the robot and environment as well as the results of testing. The chapter concludes with a discussion of the results.

\subsubsection{Experimental Setup}

The 3-DOF MRR with a rigidly attached hammer as its last link can be seen in Figure 20. Kinematic and dynamic parameters are included in Table 3. Link inertias are represented with respect to the frame rigidly attached to joint $j$. Links are rectangular prisms with a square cross-section, making the non-diagonal terms of the link inertia matrix zero. 


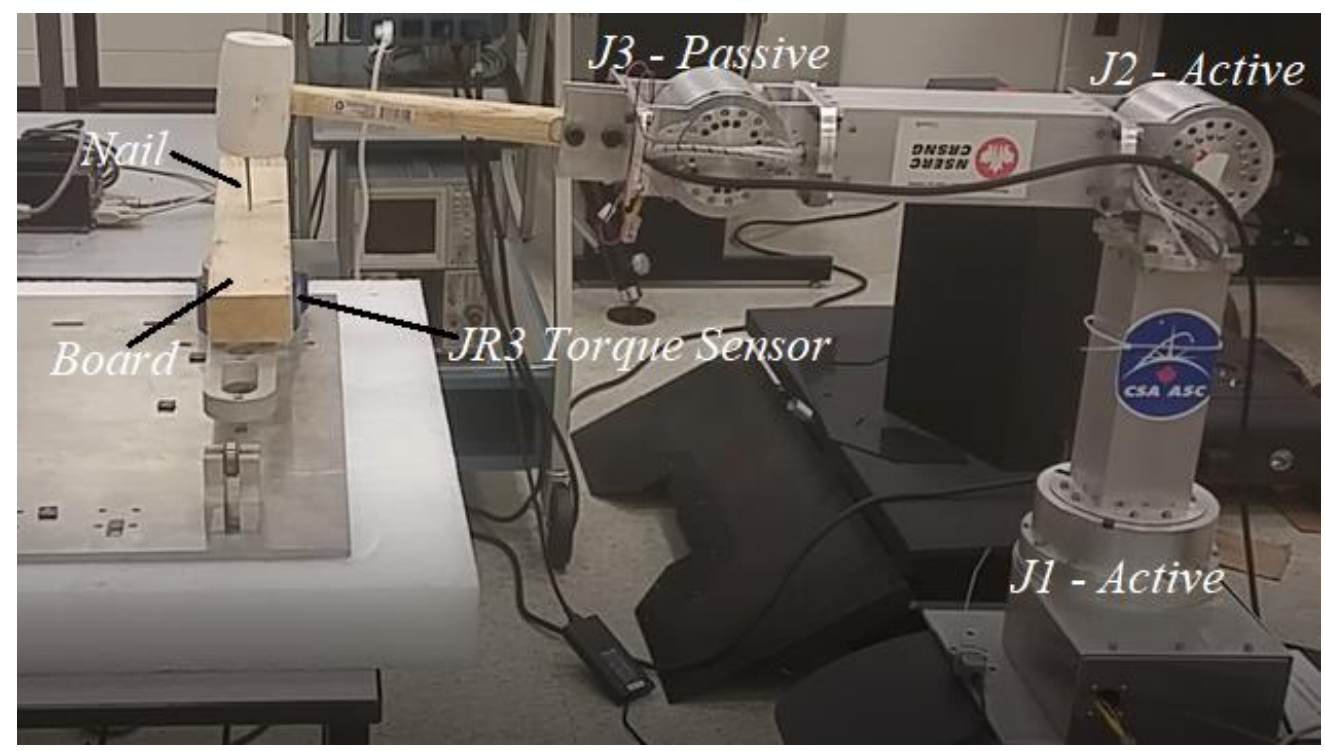

FigURE 20 DiAgRAM OF MRR WITH HAMMER END-EFFECTOR

TABLE 3 MRR LiNK DYNAMIC PARAMETERS

\begin{tabular}{|c|c|c|c|}
\hline & Link 1 & Link 2 & Link 3 \\
\hline Link Length $(\boldsymbol{m})$ & 0.48 & 0.38 & 0.38 \\
C.G. Location $(\boldsymbol{m})$ & 0.24 & 0.19 & 0.35 \\
\hline Link mass $(\boldsymbol{k g})$ & 3.5 & 3.5 & 1.0 \\
\hline Inertia $\left(\boldsymbol{k g} \boldsymbol{m}^{\mathbf{2}}\right)$ & 0.25 & & 0.017 \\
$\boldsymbol{I}_{\boldsymbol{x} \boldsymbol{j}}^{\boldsymbol{j}}$ & 0.017 & 0.017 & 0.25 \\
$\boldsymbol{I}_{\boldsymbol{y} \boldsymbol{j}}$ & 0.25 & 0.25 & 0.25 \\
$\boldsymbol{I}_{\mathbf{z z}}^{\boldsymbol{j}}$ & 133 & 0.25 & 101 \\
\hline Gear Ratio & & 101 & \\
\hline
\end{tabular}

Limits for the impulse and impact velocity for the harmonic drives equipped on each joint are obtained from the manufacturer manual [50] and are recorded in Table 4. 
TABle 4 Harmonic Drive Limit Torque Ratings [50]

\begin{tabular}{|l|c|c|c|c|c|c|}
\hline & $\begin{array}{c}\text { Rated } \\
\text { Torque } \\
(\mathbf{N m})\end{array}$ & $\begin{array}{c}\text { Repeated } \\
\text { Peak Torque } \\
(\mathbf{N m})\end{array}$ & $\begin{array}{c}\text { Momentary } \\
\text { Peak Torque } \\
(\mathbf{N m})\end{array}$ & $\begin{array}{c}\text { Safest } \\
\text { Link } \\
\text { RPM at } \\
\text { Impact }\end{array}$ & $\begin{array}{c}\text { Avg. } \\
\text { Link } \\
\text { RPM }\end{array}$ & $\begin{array}{c}\text { Max. } \\
\text { Link } \\
\text { RPM }\end{array}$ \\
\hline SHD17 & 16 & 37 & 71 & 5.94 & 8.51 & 19.8 \\
\hline SHD25 & 47 & 110 & 184 & 4.51 & 6.46 & 15.0 \\
\hline
\end{tabular}

The hammer was chosen to have the lowest weight and a large surface in order to reduce effects of position control error. A rubber head also reduces the likelihood of damage to the robot in case of any other emergency. The "Safe Link RPM" entry in Table 4 is the link RPM calculated using equation (56) by setting the left-hand side to its maximum value of $1 \times 10^{4}$, representing the number of impacts during which the Momentary Peak Torque value is surpassed. This is a very safe limit for the hammering task which guarantees safety for the robot during experimentation. The link RPM can be higher, limited by the maximum input speed of the motor as on the rightmost entry of the table.

External impulsive force data is collected using the JR3 6-DOF force/torque sensor with analog signal collected by Quanser Q8 DAQ system as in Figure 21 where up is positive such that experimental data will display a negative sign. The same experiment is repeated on two different materials and the effective mass is calculated based on the nail penetration, time of impact, and the integral of the F/T signal required by equations (33) and (34). 


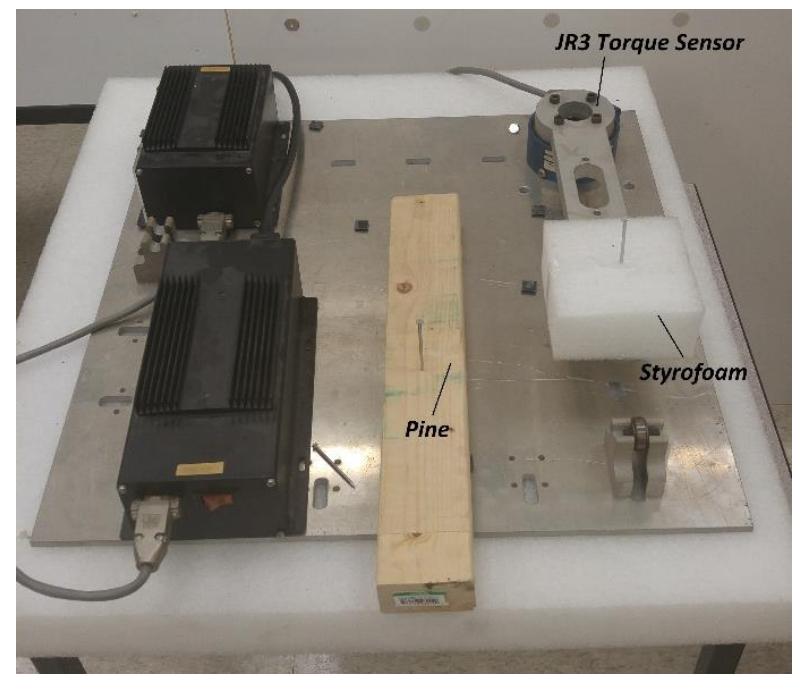

Figure 21 NAIL/BOARD ENVIRONMENT AND F/T SENSOR SET-UP

The experiment procedure shown in Figure 22(a) shows the robot in its initial position with joint 2 acting in position control to move the hammer towards the nail as in Figure 22(b). The impact occurs in the "home" configuration of the robot as seen in Figure 22(c).

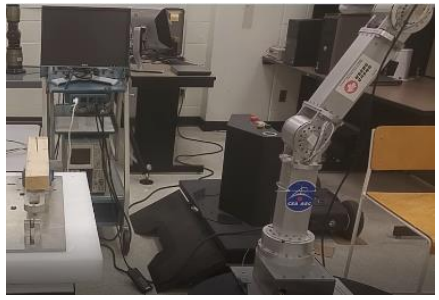

(a)

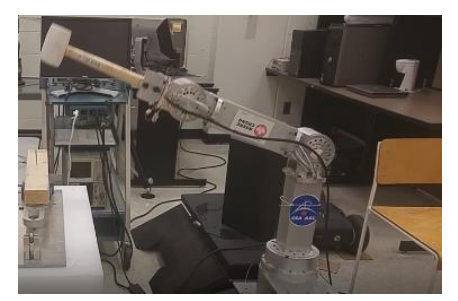

(b)

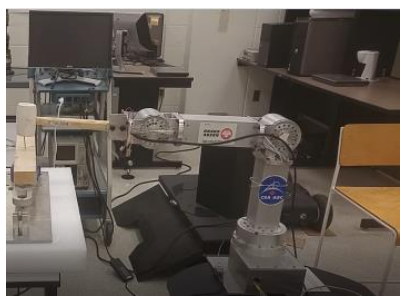

(c)

Figure 22 Experiment Procedure

The first simulation of the collision was conducted offline with the assumption that the nail will not penetrate the material. In other words, the effective mass, $\left[M_{\text {nail }}^{*}\right]=\infty$. The worst-case scenario is that the collision causes a maximum restitution impulse, therefore the 
coefficient of restitution, $e=1$. Although there are more efficient configurations for hammering, the chosen final configuration was set to its home location of zero to simplify the procedure. The robot works in position control and trajectories are polynomial trajectories in joint space. Results of the simulation are recorded in Table 5.

TABLE 5 COLLISION SIMULATION RESULTS (UNADJUSTED)

\begin{tabular}{|c|c|c|c|}
\hline & $\begin{array}{l}\text { External impulse, } \\
\qquad \widehat{\boldsymbol{F}}_{\text {ext }},(\boldsymbol{N s})\end{array}$ & $\begin{array}{c}\text { Link velocity change, } \\
\qquad \boldsymbol{\Delta q},(\boldsymbol{r a d} / \mathbf{s})\end{array}$ & $\begin{array}{l}\text { Internal impulse, } \hat{\boldsymbol{\tau}}, \\
\qquad(\boldsymbol{N} \boldsymbol{s})\end{array}$ \\
\hline $\begin{array}{c}\text { Simulation } \\
\text { Results }\end{array}$ & {$\left[\begin{array}{c}\hat{F}_{x} \\
\hat{F}_{y} \\
\hat{F}_{z} \\
\hat{\tau}_{x} \\
\hat{\tau}_{y} \\
\hat{\tau}_{z}\end{array}\right]=\left[\begin{array}{c}0 \\
0 \\
-2.06 \\
0 \\
0 \\
0\end{array}\right]$} & {$\left[\begin{array}{l}\Delta \dot{q}_{1} \\
\Delta \dot{q}_{2} \\
\Delta \dot{q}_{3}\end{array}\right]=\left[\begin{array}{c}0.0 \\
-0.98 \\
-0.72\end{array}\right]$} & {$\left[\begin{array}{l}\hat{\tau}_{y 1} \\
\hat{\tau}_{z 2} \\
\hat{\tau}_{z 3}\end{array}\right]=\left[\begin{array}{c}0.0 \\
0.84 \\
0.72\end{array}\right]$} \\
\hline
\end{tabular}

Note that with the assumed impact duration of $t_{s}=50 \mathrm{~ms}$, equation (58) prescribes a limit of $\hat{\tau}<17 \cdot 50 \times 10^{-3}<0.85 \mathrm{Ns}$, which means that all the internal impulses are below the rated torque limit.

\subsubsection{Results}

\subsubsection{External Impulse and Effective Mass}

Two test runs were performed for both Styrofoam and hardwood. Each test run consisted of four hammer swings. The force impulse on the JR3 F/T sensor Z-direction in a Styrofoam and hardwood test can be seen in Figure 23 and Figure 24. Each swing profile was 
integrated using trapezoidal approximation to determine the total external impulse during the collision.
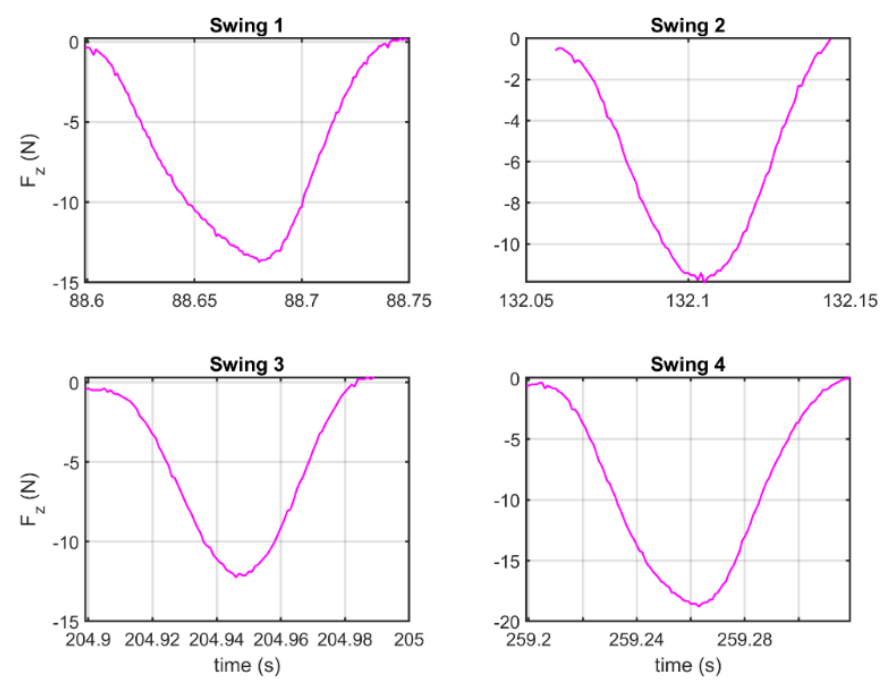

FIGURE 23 EXTERNAL FORCE DATA FOR A STYROFOAM TEST
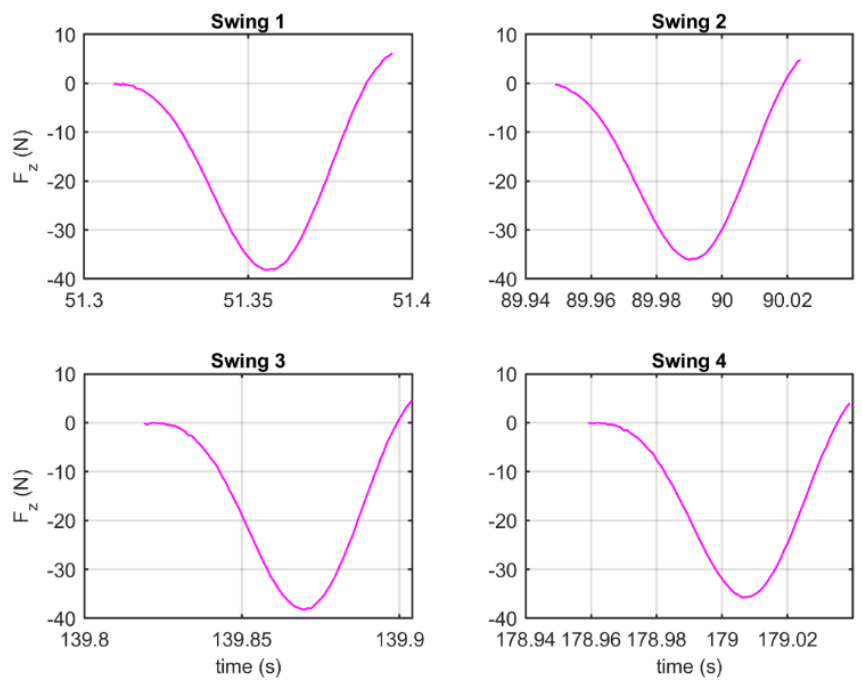

FIGURE 24 EXTERNAL FORCE DATA FOR A HARDWOOD TEST 
The initial nail height was $40 \mathrm{~mm}$ and the penetration after each swing was recorded in Table 6 for the first test, and Table 7 for the second. From the data it is clear that higher nail penetration corresponded to longer impact duration and larger external impulse. However, the same effect can be explained by looking at calculated effective mass. Lower effective mass corresponds to more penetration, which makes sense since that implies less hardness of the material. As the nail went in deeper into the material, the effective mass increased. This is likely because of higher resistance due to friction between the material and nail surface. Swing 4 in Table 6 shows higher penetration because the reference position for joint 2 was changed for this swing mid-test particularly because the hammer was only marginally reaching the nail head. The data is still useful as each swing can be treated separately. There is a maximum limit to how much the reference position can be changed to produce more nail penetration. Selecting that maximum value (corresponding to the position of the board material surface) and performing all swings in that way is ideal.

\section{TABLE 6 EXTERNAL F/T SENSOR STYROFOAM TEST 1 RESULTS}

\begin{tabular}{|c|c|c|c|c|}
\hline \multirow[b]{2}{*}{ Swing \# } & \multicolumn{4}{|c|}{ Styrofoam Test 1} \\
\hline & 1 & 2 & 3 & 4 \\
\hline $\begin{array}{c}\text { Nail } \\
\text { penetration } \\
(\mathrm{mm})\end{array}$ & 22 & 4 & 3 & 11 \\
\hline $\begin{array}{c}\text { Impact } \\
\text { duration, } \\
t_{s}(m s)\end{array}$ & 150 & 85 & 90 & 120 \\
\hline External & -1.02 & -0.53 & -0.49 & -1.05 \\
\hline
\end{tabular}




\begin{tabular}{|c|c|c|c|c|}
\hline $\begin{array}{l}\text { impulse, } \\
\widehat{F}_{\text {ext }}(N s)\end{array}$ & & & & \\
\hline $\begin{array}{c}\text { Effective mass, } \\
{\left[M_{\text {nail }}^{*}\right](\boldsymbol{k g})}\end{array}$ & 7.02 & 11.2 & 14.6 & 11.5 \\
\hline
\end{tabular}

TABLE 7 EXTERNAL F/T SENSOR STYROFOAM TEST 2 RESUlTS

\begin{tabular}{|c|c|c|c|c|}
\hline \multirow[b]{2}{*}{ Swing \# } & \multicolumn{4}{|c|}{ Styrofoam Test 2} \\
\hline & 1 & 2 & 3 & 4 \\
\hline $\begin{array}{c}\text { Nail } \\
\text { penetration } \\
(m m)\end{array}$ & 20 & 13 & 5 & 2 \\
\hline $\begin{array}{c}\text { Impact } \\
\text { duration, } \\
t_{s}(\mathrm{~m} s)\end{array}$ & 140 & 130 & 90 & 90 \\
\hline $\begin{array}{l}\text { External } \\
\text { impulse, } \\
\widehat{F}_{\text {ext }}(N s)\end{array}$ & -0.95 & -1.08 & -0.67 & -0.76 \\
\hline $\begin{array}{c}\text { Effective mass, } \\
{\left[M_{\text {nail }}^{*}\right](\boldsymbol{k g})}\end{array}$ & 6.7 & 10.9 & 12.1 & 34.1 \\
\hline
\end{tabular}

Because the hardwood was never penetrated, only data relating to the impact duration and external impulse was collected from the JR3 F/T sensor as in Table 8. The effective mass for each swing is thus infinity. Because each swing was performed with no difference in selectable parameters, duration of impact and external impulse are extremely similar through each test. The discrepancies in external impulse from swing to swing can be explained by position error of the joints. Consider the position error of joint 2 during the hardwood test as in Figure 25 which fluctuated slightly in each swing. Joint 3 position error was set to zero for each swing for the joint to remain in passive mode, but after each swing the joint slightly 
shifted which required active position control to bring it back to the reference position of zero degrees. During these moments when the joint 3 was in position control, there was also a slight position error, so the swings were only marginally different initial position, but this does reflect in the force data.

TABLE 8 EXTERNAL F/T SENSOR HARDWOOD TEST RESULTS

\begin{tabular}{|c|c|c|c|c|}
\hline \multirow[b]{2}{*}{ Swing \# } & \multicolumn{4}{|c|}{ Hardwood Test 1} \\
\hline & 1 & 2 & 3 & 4 \\
\hline $\begin{array}{c}\text { Impact } \\
\text { duration, } \\
t_{s}(m s)\end{array}$ & 75 & 70 & 80 & 80 \\
\hline $\begin{array}{c}\text { External } \\
\text { impulse, } \\
\widehat{F}_{\text {ext }}(N s)\end{array}$ & -1.79 & -1.3 & -1.29 & -1.34 \\
\hline
\end{tabular}

\begin{tabular}{|c|c|c|c|c|}
\hline \multirow{2}{*}{$\begin{array}{c}\text { Impact } \\
\text { duration, } \\
t_{s}(m s)\end{array}$} & \multicolumn{4}{|c|}{ Hardwood Test 2} \\
\hline & 85 & 75 & 85 & 80 \\
\hline $\begin{array}{l}\text { External } \\
\text { impulse, } \\
\widehat{F}_{\text {ext }}(N s)\end{array}$ & -1.41 & -1.28 & -1.42 & -1.26 \\
\hline
\end{tabular}




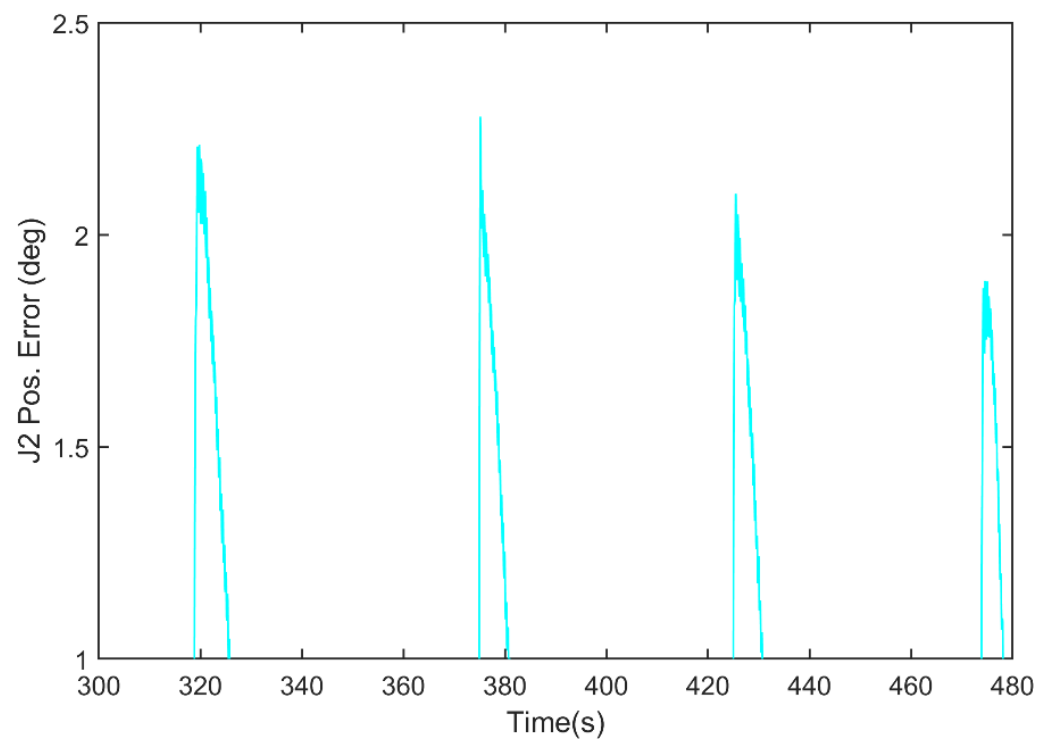

FIGURE 25 JOINT 2 POSITION ERROR DURING HARDWOOD TEST (ACTIVE MODE)

To determine the accuracy and precision of the external impulse model, the same simulation used to produce results in Table 5 was conducted with adjusted values for the coefficient of restitution, $e$, and effective mass, $\left[M_{\text {nail }}^{*}\right]$. First, however, this requires approximating the values of the coefficient of restitution for the collision. The definition of $e$ per the derivation in Chapter 2 of the thesis comes from [48] which states that:

$$
e=\frac{\left(v_{B}\right)_{2}-\left(v_{A}\right)_{2}}{\left(v_{A}\right)_{1}-\left(v_{B}\right)_{1}}
$$

where Figure 5 illustrates the scenario of the two bodies colliding. If the nail mass is considered to be negligible compared to the board such that neither is considered to have developed any velocity after collision, then both $\left(v_{B}\right)_{1}$ and $\left(v_{B}\right)_{2}$ are approximately zero. 
While this is not exactly true, it is close enough to provide a working approximation of $e$. Since it is assumed that the collision occurs over a small enough period of time that the joint position vector, $q$, has not changed very much, the end-effector velocity in the z-direction both before and after impact may be calculated using the geometric Jacobian, $v_{E}=J_{H} \dot{q}$ given the vectors $\dot{q}_{1}$ and $\dot{q}_{2}$ representing the respective joint velocities. The post-impact joint velocity was measured using an encoder on each joint as seen in Figure 27. Figure 26 shows a comparison between the model predictions of external impulse for a few swings with values for coefficient of restitution and effective mass as shown. For the hardwood tests, the effective mass remained at infinity, only the value for $e$ was adjusted. The external force estimates are similar in prediction and accuracy as reported in past literature [35]. More importantly, the encoder data in Figure 27 can be used to evaluate the effectiveness of the passive mode to reduce stress on the joint. The following chapter includes a discussion on the topic. 


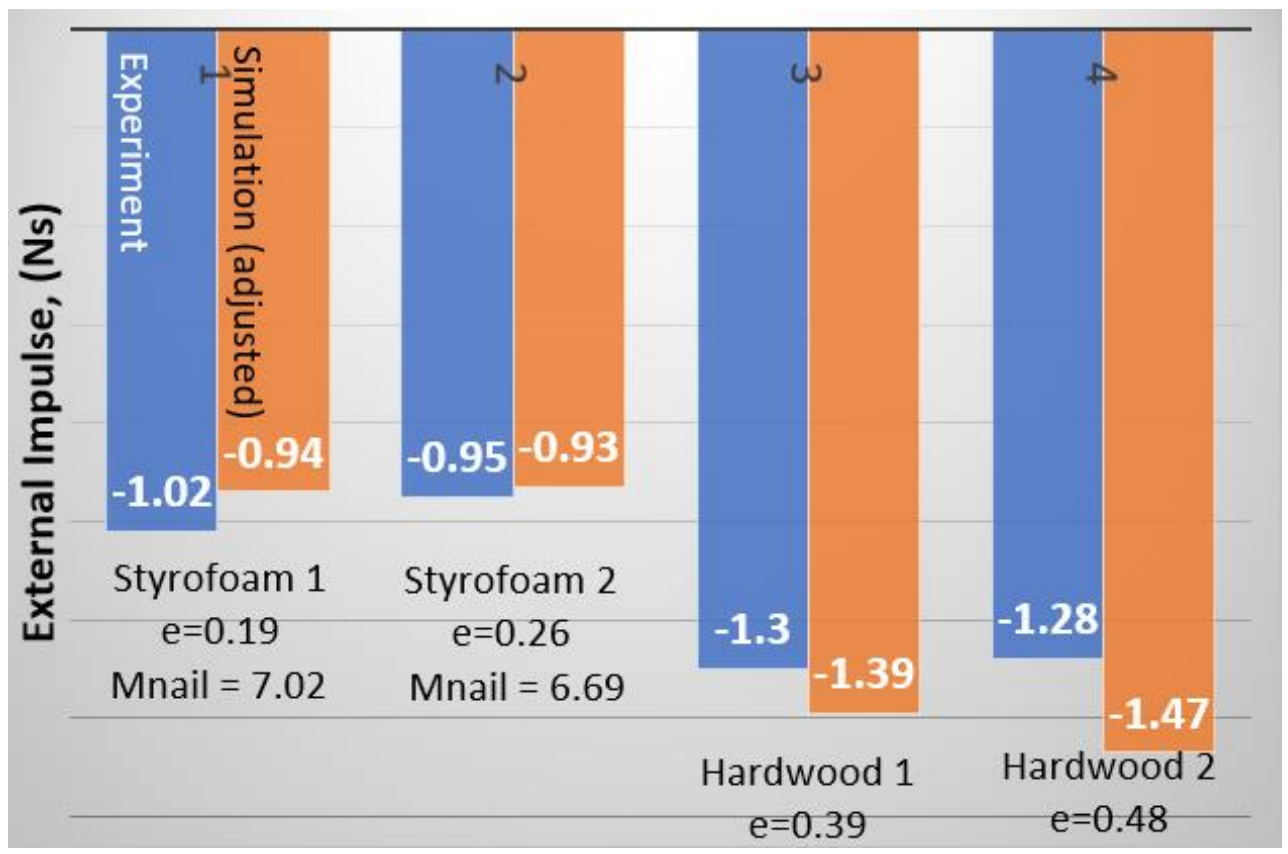

FIGURE 26 TEST AND SIMULATION EXTERNAL IMPULSE COMPARISON
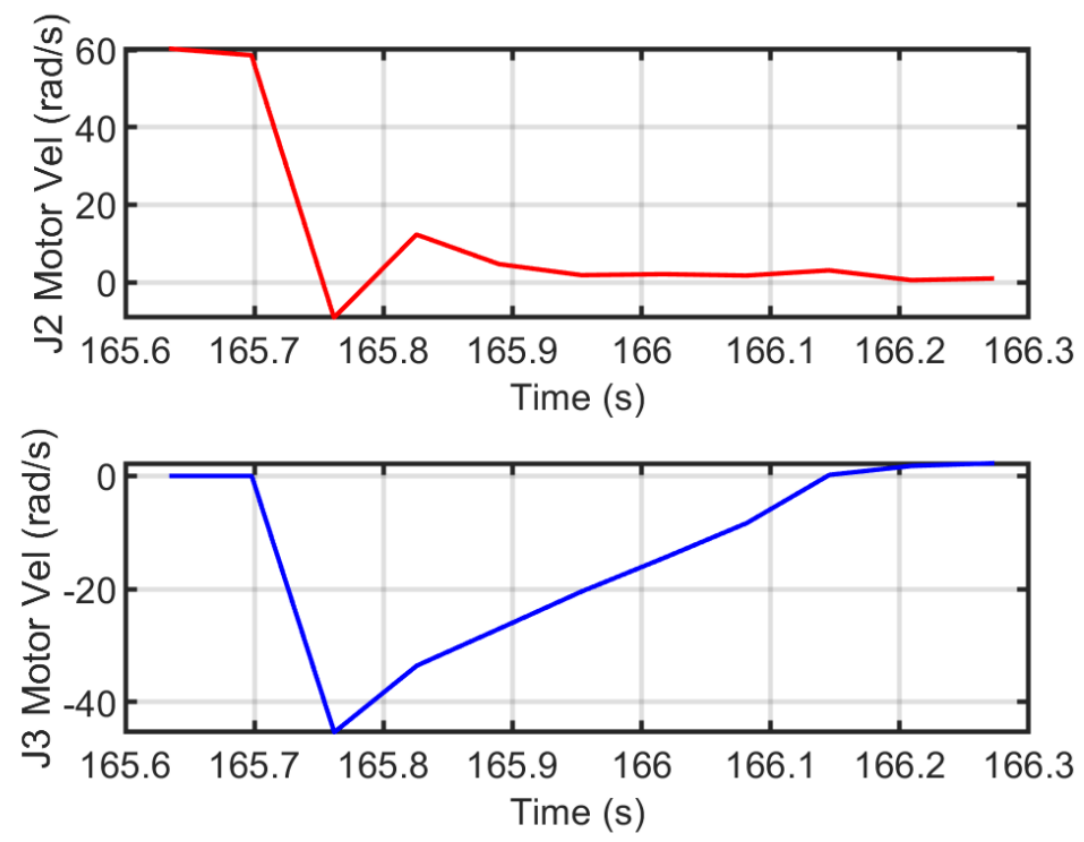

FIGURE 27 MOTOR VELOCITY DURING HARDWOOD 2 TEST 


\subsubsection{Internal Impulse and Passive Mode}

As mentioned earlier, the lack of internal joint torque sensing means that it is not possible to do the sort of comparisons and verifications of internal impulse model as with the external impulse model. However, one important portion of the model can be seen in (31) and (41) which includes changes in joint velocities due to impact. Because the encoder measurements are available, they can be compared against model predictions. The same data also reveals the difference in behavior of the joint in active mode and in passive mode.

Consider Figure 27 where the red line (top) shows the motor velocity of joint 2 . The motor is connected to the shaft of the harmonic drive and acts as an input. Right after the collision happens, the joint motor velocity reduces greatly and falls below the zero line. A few milliseconds later, the motor attempted to bring itself back to its reference velocity that was requested by the supervisory controller. For a short time, the link was moving in the opposite direction from the motor, causing them to twist the shaft and thus the bearing of the harmonic drive in opposite direction which, combined, causes a very high stress on two of the most sensitive components of a harmonic drive. The blue line (bottom), on the other hand, shows joint 3 motor velocity in passive mode which had a sudden increase and slowly came to a stop. Based on the reaction seen in joint 2, it is reasonable to conclude that if joint 3 were also set into active mode the motor reaction would have been to twist the harmonic drive shaft until the link finds its reference position. Setting the joint into passive mode successfully avoids stressing the harmonic drive bearing and motor which is an important discovery of this experimental study. 
Before concluding, an analysis of the joint velocities for the same tests and simulation as in Figure 26 is conducted. The internal joint model assumes that the joint fully restrains the impact loads, but for a freely moving joint, it is assumed to almost instantaneously reach a finite velocity according to (31). For a given value of $e$, the simulation provides maximum values for joint velocities since free and frictionless joints, even with friction compensation, do not exist. Figure 28 shows simulation and experiment values.

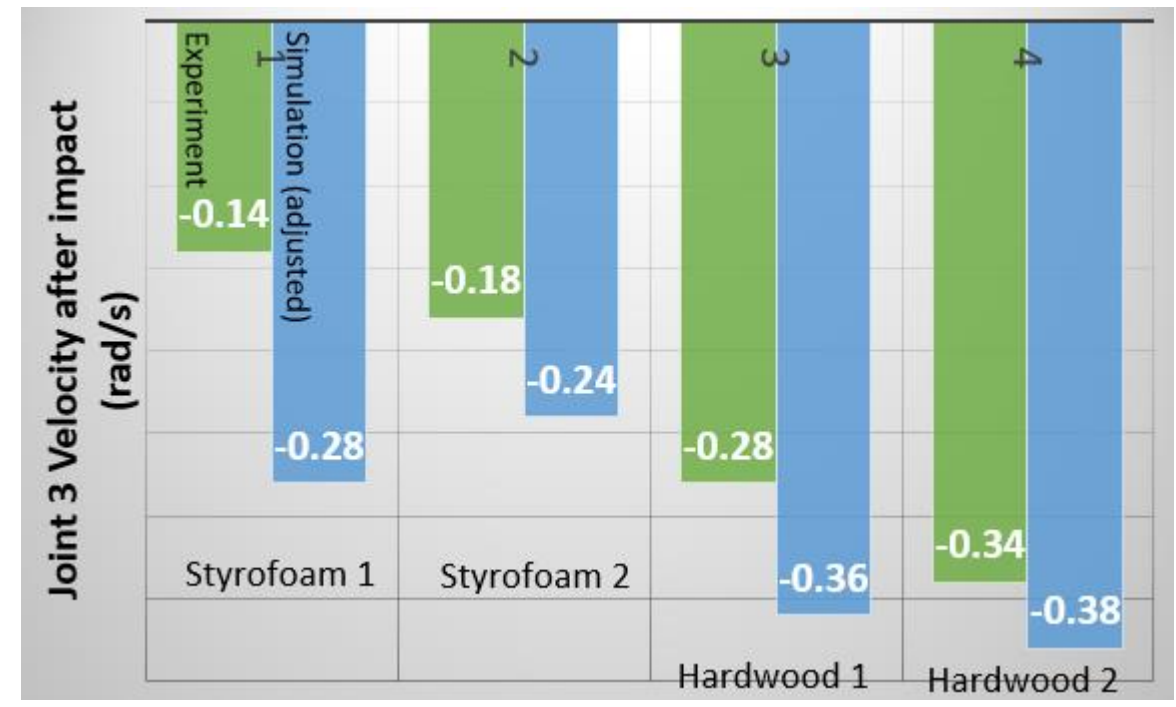

FIGURE 28 FRICTIONLESS JOINT VELOCITY AS COMPARED TO TEST VALUES

The green bars (left) indicating experimental results are always below the predicted change in joint velocities, indicating conservative estimates. The discrepancy is likely due to uncompensated joint friction. Because friction is a function of both position and velocity with a higher starting friction as in Figure 15, it is expected to see a better prediction for collision with hardwood that generated larger external impulse. After the initial peak of friction is surpassed, it is a lot easier for the joint to be driven, but in a collision with Styrofoam, that 
barrier may not necessarily be exceeded and thus the test results show a large discrepancy from predicted values. It is clear from (53) that the internal joint torques are proportional to the magnitudes of entries in the $[D]$ matrix. Large changes in joint velocities are likely to be related to higher internal joint torques and since none of the experimental values have exceeded the predicted values, it is likely that the internal joint impulses also remained below the limit specified by the rated torque.

\subsection{Concluding Remarks}

Chapter 5 combines the knowledge and ideas that have been compiled in all the earlier chapters and presents a step-by-step strategy for any striking task including hammering that ensure integrity of MRR joints for the longest possible life duration. This is the main contribution of the thesis as well the experimental study showing the predictive capabilities of impulse models and effectiveness of passive mode in reducing stress on the harmonic drive. 


\section{CONCLUSIONS AND FUTURE WORKS}

The main work presented in this thesis is the development of a hammering strategy using the multiple working mode approach. The presented strategy solves the problem of joint torque build-up in a position controlled joint during impact. To do so, the joint is switched to passive mode such that the motor does not twist on the harmonic drive shaft in an attempt to reach a reference trajectory sent by the supervisory controller. Analytical modelling of external and internal joint torques function as criteria to determine whether a joint should be switched to passive mode. For real-time mode switching, such that the necessary joints may be switched quickly into passive mode, two extra criteria are proposed. Simulations and an experimental study show the analytic model capabilities to predict impulses and effectiveness of the passive mode to reduce stress on the joint.

While robot hammering was studied in this work specifically, the approach can generally be applied to any collision or impact of an end-effector with an environment of unknown mass or hardness. The simulation of the entire collision can be performed either offline or online which provides versatility. Performing them online may be useful in a robot made specifically for striking tasks and with some optimization it can become an efficient striker. If the goal is to perform a variety of maintenance tasks, then the simulation may be performed offline to save on computational space and power.

Some limitations of this method include the fact that it is assumed the robot is able to develop the desired end-effector velocities within the space that is available around it. In 
experiments, the robot had to start its trajectory away from its home position so that it can accelerate to the desired final velocity. Physical space around the robot may be a factor. Next, the effective mass of the environment may not be estimated without performing a test ahead of time and specifying it in simulations. A robot would have to be equipped with some sort of method to estimate penetration of the nail with each hit to use the effective mass model online, making it difficult to optimize the strategy with a lot of unknowns about the environment.

While the experiment in this work showed the effectiveness of passive mode, it was done so without friction compensation (and joint torque sensing). A study of the internal impulse model should be conducted to determine if the estimates are accurate at the same time. The proposed real-time mode switching criteria can also be investigated so that joints may stay in active mode for position control until exactly the moment when it should switch to passive mode. 


\section{APPENDIX}

\section{Appendix 1 Impact Simulation Code}

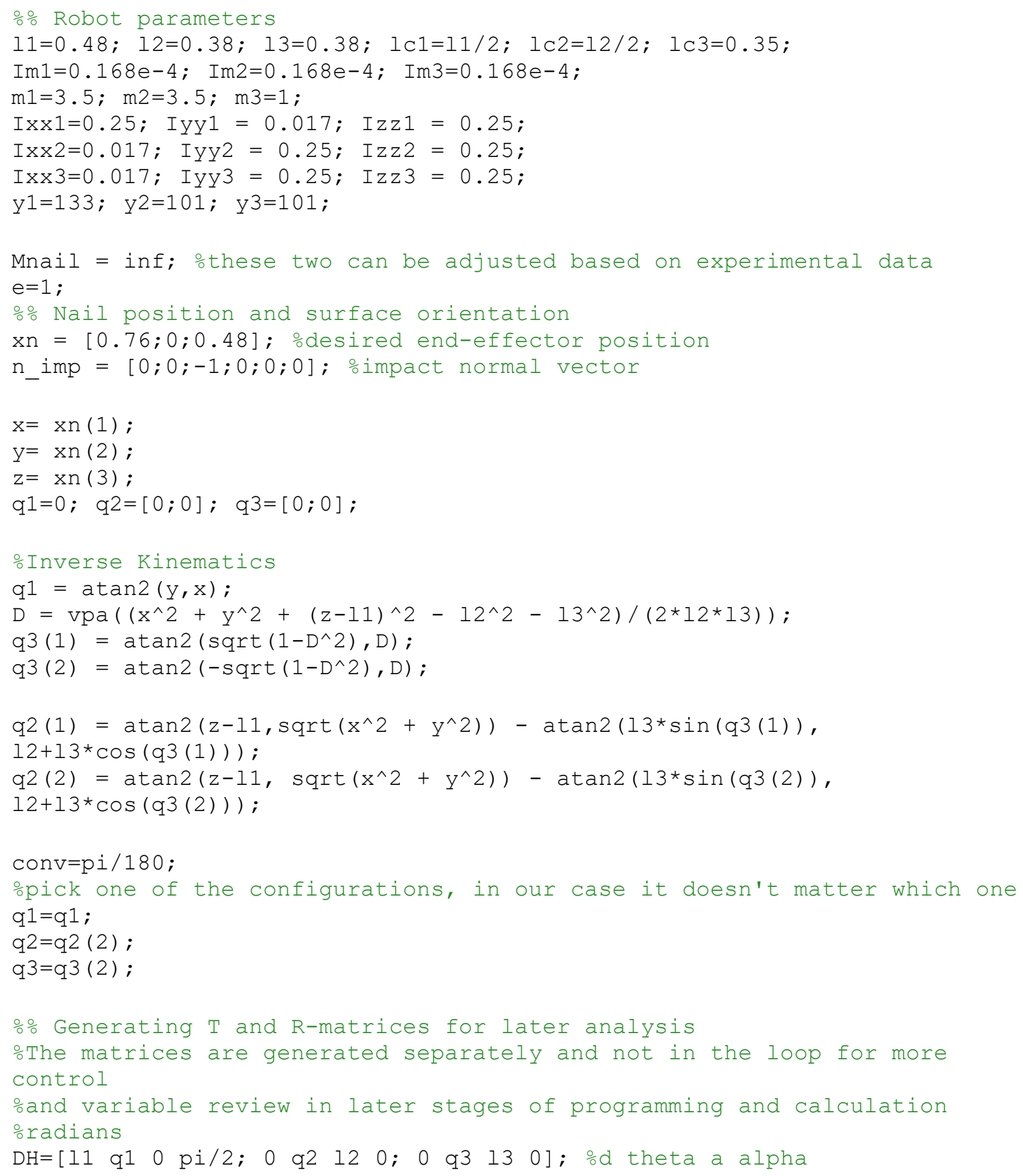




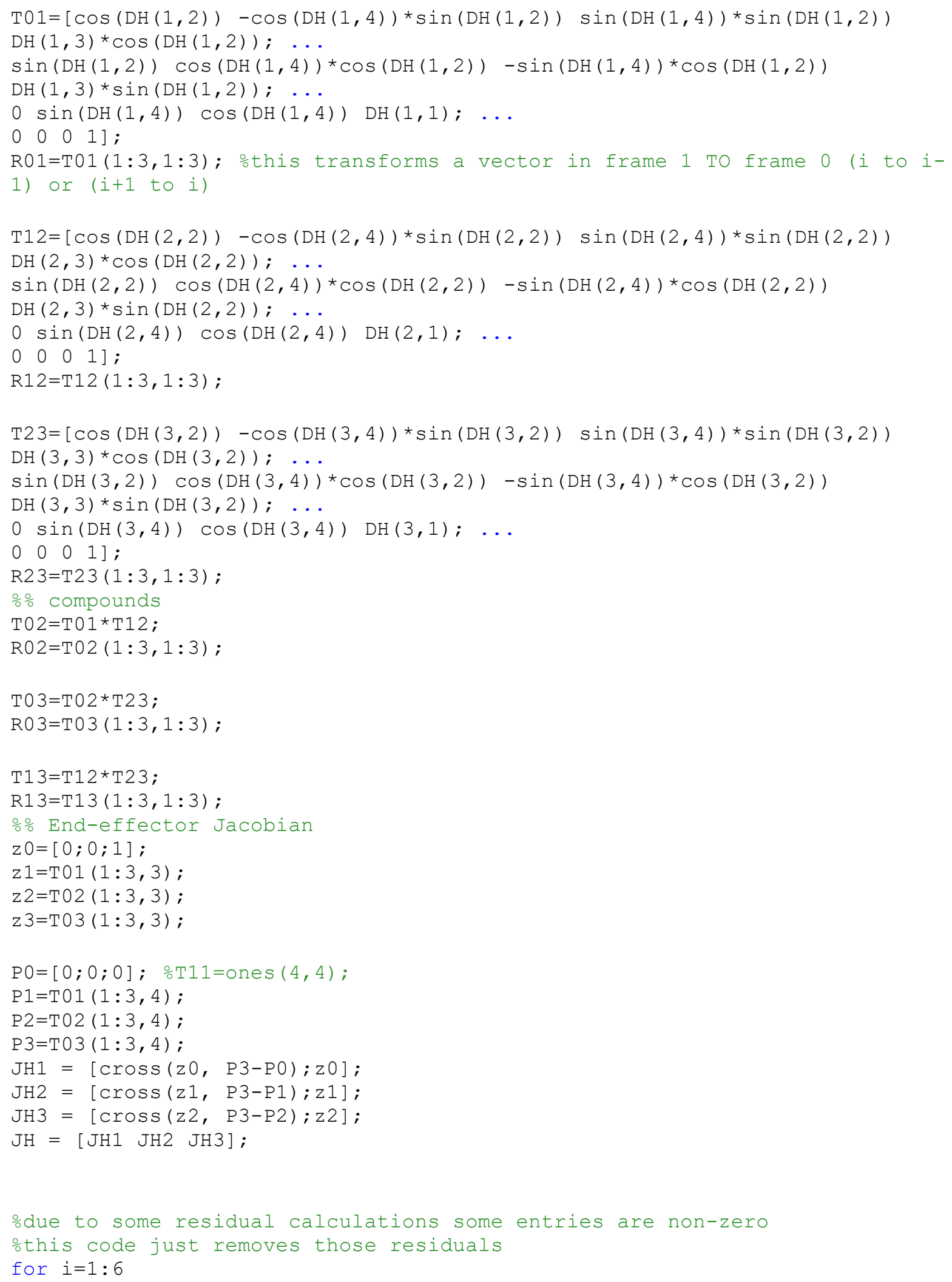




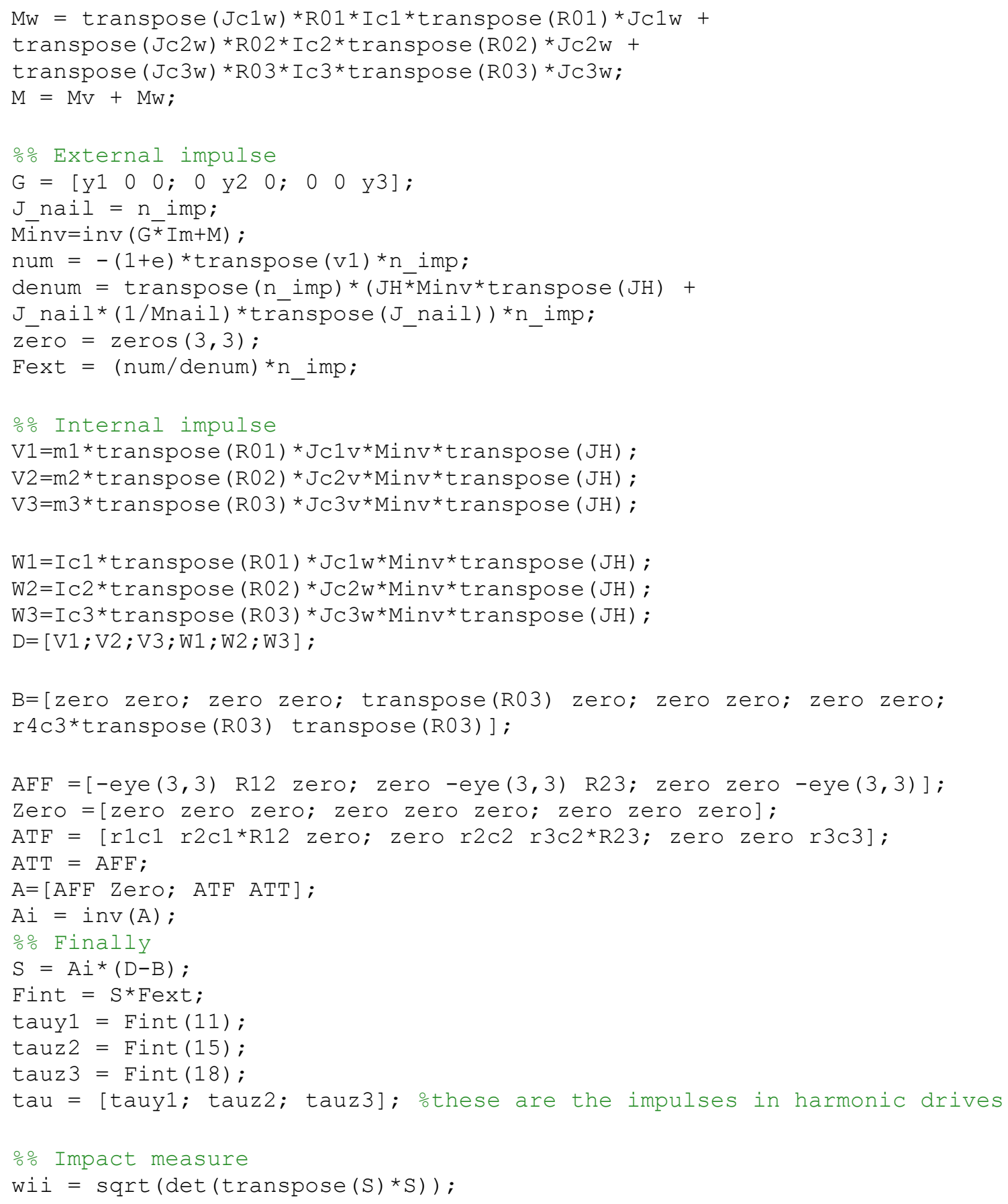




\section{REFERENCES}

[1] M. W. Spong, F. L. Lewis and C. T. Abdallah, Robot Control: Dynamics, Motion Planning, and Analysis, New York: Institute of Electrical and Electronics Engineers, 1993.

[2] A. De Luca and L. Ferrajoli, "Exploiting Robot Redundancy in Collision Detection and Reaction," in IEEE/RSJ International Conference on Intelligent Robots and Systems (IROS), Nice, 2008.

[3] A. De Luca, A. Albu-Schaffer, S. Haddadin and G. Hirzinger, "Collision Detection and Safe Reaction with the DLR-III Lightweight Manipulator Arm," in IEEE International Conference on Intelligent Robots and Systems (IROS), Beijin, 2006.

[4] M. Grebenstein, A. Albu-Schaffer, T. Bahls, M. Chalon, O. Eiberger, W. Friedl, R. Gruber, S. Haddadin, U. Hagn, R. Haslinger, H. Hoppner, S. Jorg, M. Nickl, A. Nothhelfer, F. Petit, J. Reill, N. Seitz, T. Wimbock, S. Wolf, T. Wusthoff and G. Hirzinger, "The DLR Hand Arm System," in IEEE International Conference on Robotics and Automation (ICRA), Shanghai, 2011.

[5] Y. Sakagami, Watanabe Ryujin, C. Aoyama, S. Matsunaga, N. Higaki and K. Fujimura, "The intelligent ASIMO: System Overview and Integration," in IEEE/RSJ International Conference on Intelligent Robots and Systems (IROS), Lausanne, 2002.

[6] Z. Qian and D. Zhang, "Impact Dynamics of Multi-link Robots with Link and Joint Flexibility," Applied Mechanics and Materials, Vols. 226-228, pp. 685-692, 2012.

[7] J. Vogel, N. Takemura, H. Hoppner, P. van der Smagt and G. Ganesh, "Hitting the Sweet Spot: Automatic Optimization of Energy Transfer during Tool-held Hits," in IEEE International Conference on Robotics and Automation (ICRA), Singapore, 2017.

[8] D. N. Nenchev and K. Yoshida, "Impact Analysis and Post-Impact Motion Control Issues of a Free-Floating Space Robot Subject to a Force Impulse," IEEE Transactions on Robotics and Automation, vol. 15, no. 3, pp. 548-557, 1999.

[9] B. Bauml, T. Wimbock and G. Hirzinger, "Kinematically Optimal Catching a Flying Ball with a Hand-Arm-System," in IEEE/RSJ International Conference on Intelligent Robots and Systems (IROS), Taipei, 2010.

[10] H. Schempf, C. Kraeuter and M. Blackwell, "Roboleg: A Robotic Soccer-ball Kicking Leg," in IEEE International Conference on Robotics and Automation (ICRA), Nagoya, 1995.

[11] T. Izumi and Y. Hitaka, "Hitting from Any Direction in 3-D Space by a Robot with a Flexible Link Hammer," IEEE Transactions on Robotics and Automation, vol. 13, no. 2, pp. 296-301, 1997.

[12] X.-Z. Zheng, W. Inamura, K. Shibata and K. Ito, "A Learning and Dynamic Pattern Generating Architecture for Skillful Robotic Baseball Batting System," in IEEE International Conference on Robotics and Automation (ICRA), San Francisco, 2000.

[13] M. Garabini, A. Passaglia, F. Belo, P. Salaris and A. Bicchi, "Optimality Principles in Variable Stiffness Control: The VSA Hammer," in IEEE/RSJ International Conference on Intelligent Robots and Systems (IROS), San Francisco, 2011.

[14] M. Aiple and A. Schiele, "Towards Teleoperation with Human-like Dynamics: Human Use of Elastic Tools," in IEEE World Haptics Conference (WHC), Munich, 2017.

[15] N. Hogan, "Impedance Control: An Approach to Manipulation," in American Control 
Conference, San Diego, 1984.

[16] G. A. Pratt and M. M. Williamson, "Series Elastic Actuator," in IEEE/RSJ International Conference on Intelligent Robots and Systems (IROS), Pittsburgh, 1995.

[17] S. Wolf, O. Eiberger and G. Hirzinger, "The DLR FSJ: Energy Based Design of a Variable Stiffness Joint," in IEEE International Conference on Robotics and Automation (ICRA), Shanghai, 2011.

[18] G. Grioli, S. Wolf, M. Garabini, M. Catalano, E. Burdet, D. Caldwell, R. Carloni, W. Friedl, M. Grebenstein, M. Laffranchi, D. Lefeber, S. Stramigioli, N. Tsagarakis, M. van Damme, B. Vanderborght, A. Albu-Schaeffer and A. Bicchi, "Variable Stiffness Actuators: The User's Point of View," The International Journal of Robotics Research, vol. 34, no. 6, pp. 727-743, 2015.

[19] S. Wolf, G. Grioli, O. Eiberger, W. Friedl, M. Grebenstein, H. Hoppner, E. Burdet, D. G. Caldwell, R. Carloni, M. G. Catalano, D. Lefeber, S. Stramigioli, N. Tsagarakis, M. Van Damme, R. Van Ham, B. Vanderborght, L. C. Visser, A. Bicchi and A. Albu-Schaffer, "Variable Stiffness Actuators: Review on Design and Components," IEEE/ASME Transactions on Mechatronics, vol. 21, no. 5, pp. 2418-2430, 2016.

[20] A. De Luca and R. Mattone, "Sensorless Robot Collision Detection and Hybrid Force/Motion Control," in IEEE International Conference on Robotics and Automation (ICRA), Barcelona, 2005.

[21] B. Hove and J.-J. E. Slotine, "Experiments in Robotic Catching," in American Control Conference, Boston, 1991.

[22] D. Zhang and S. F. Zhou, "Dynamic Analysis of Flexible-link and Flexible-joint Robots," Applies Mathematics and Mechanics, vol. 27, no. 5, p. 695, 2006.

[23] S. Wang, G. Cheng, X. Chen and J. Yang, "Natural Frequency Analysis and Experiment for 3SPS+1PS Parallel Hip Joint Manipulator based on Rigid-flexible Coupling Theory," Journal of Mechanical Science and Technology, vol. 31, no. 3, pp. 1447-1462, 2017.

[24] S.-J. Huang and T.-Y. Wang, "Structural Dynamics Analysis of Spatial Robots with Finite Element Approach," Computers \& Structures, vol. 46, no. 4, pp. 703-716, 1993.

[25] T. Izumi and Y. Hitaka, "Control of a Flexible Link Hammer in a Gravitational Field and its Application to a Home Robot Tapping Human Shoulders," in IEEE/RSJ International Conference on Intelligent Robots and Systems (IROS), Yokohama, 1993.

[26] A. Kobayashi, H. Suzuki, T. Hirogaki and E. Aoyama, "Investigation of Impact Task Performed by Humanoid Robot Using Input Shaping Control Method," in International Conference on Control, Automation, Robotics \& Vision, Phuket, 2016.

[27] A. S. Yigit, A. G. Ulsoy and R. A. Scott, "Spring-Dashpot Models for the Dynamics of a Radially Rotating Beam with Impact," Journal of Sound and Vibration, vol. 142, no. 3, pp. 515525, 1990.

[28] B. V. Chapnik, G. R. Heppler and J. D. Aplevich, "Modeling Impact on a One-Link Flexible Robotic Arm," IEEE Transactions on Robotics and Automation, vol. 7, no. 4, pp. 479-488, 1991.

[29] A. Tornambé, "Global Regulation of a Planar Robot Arm Striking a Surface," IEEE Transactions on Automatic Control, vol. 41, no. 10, pp. 1517-1521, 1996.

[30] K. Dupree, C.-H. Liang, G. Hu and W. E. Dixon, "Adaptive Lyapunov-Based Control of a Robot and Mass-Spring System Undergoing an Impact Collision," IEEE Transactions on Systems, Man, and Cybernetics - Part B: Cybernetics, vol. 38, no. 4, pp. 1050-1061, 2008. 
[31] Y. Hitaka and T. Izumi, "Minimum Energy Driving a Flexible Link Hammer using Neural Networks," in IEEE/RSJ International Conference on Human Robot Interaction and Cooperative Robots, Pittsburgh, PA, 1995.

[32] I. D. Walker, "Impact Configurations and Measures for Kinematically Redundant and Multiple Armed Robot Systems," IEEE Transactions on Robotics and Automation, vol. 10, no. 5, pp. 670683, 1994.

[33] J. Wittenberg, Dynamics of Systems of Rigid Bodies, Stuttgart: B.G. Teubner, 1977.

[34] J. H. Lee, B.-J. Yi, S.-R. Oh and I. H. Suh, "Performance Analysis of Sawing Based on Impulse Measure and Geometry - Dublahal Arm Approach," IEEE Transactions on Robotics, vol. 21, no. 6, pp. 1230-1240, 2005.

[35] A. Imran and B.-J. Yi, "A Closed-Form Analytical Modeling of Internal Impulses With Application to Dynamic Machining Task: Biologically Inspired Dual-Arm Robotic Approach," IEEE Robotics and Automation Letters, vol. 3, no. 1, pp. 442-449, 2018.

[36] A. Imran and B.-J. Yi, "Impulse Modeling and New Impulse Measure for Human-Like ClosedChain Manipulator," IEEE Robotics and Automation Letters, vol. 1, no. 2, pp. 868-875, 2016.

[37] T. Matsumoto, A. Konno, L. Gou and M. Uchiyama, "A Humanoid Robot that Breaks Wooden Boards Applying Impulsive Force," in IEEE/RSJ International Conference on Intelligent Robots and Systems, Beijing, 2006.

[38] T. Tsujita, A. Konno, S. Komizunai, Y. Nomura, T. Owa, T. Myojin, Y. Ayaz and M. Uchiyama, "Humanoid Robot Motion Generation for Nailing Task," in IEEE/ASME International Conference on Advanced Intelligent Mechatronics, Xi'an, 2008.

[39] A. Imran and B.-J. Yi, "Impulse Modeling and Analysis of Dual Arm Hammering Task: Humanlike Manipulator," in IEEE/RSJ International Conference on Intelligent Robots and Systems (IROS), Daejeon, 2016.

[40] H. Asada and K. Ogawa, "On the Dynamic Analysis of a Manipulator and its End Effector Interacting with the Environment," in IEEE International Conference on Robotics and Automation (ICRA), Kyoto, 1987.

[41] A. Bicchi and G. Tonietti, "Dealing with the Safety-Performance Tradeoff in Robot Arms Design and Control," IEEE Robotics and Automatin Magazine, pp. 22-33, 2004.

[42] A. Bicchi, G. Tonietti and R. Schiavi, "Safe and Fast Actuators for Machines Interacting with Humans," in IEEE Conference on Robotics and Automation, Minato-ku, 2004.

[43] F. Petit, M. Chalon, W. Friedl, M. Grebenstein, A. Albu-Schaffer and G. Hirzinger, "Bidirectional Antagonistic Variable Stiffness Actuation: Analysis, Design \& Implementation," in IEEE International Conference on Robotics and Automation (ICRA), Anchorage, 2010.

[44] P. Cherelle, A. Matthys, V. Grosu, B. Vanderborght and D. Lefeber, "The AMP-Foot 2.0: Mimicking Intact Ankle Behavior with a Powered Transtibial Prosthesis," in IEEE RAS/EMBS International Conference on Biomedical Robotics and Biomechatronics, Roma, 2012.

[45] M. Yim, W.-M. Shen, B. Salemi, D. Rus, M. Moll, H. Lipson, E. Klavins and G. S. Chirikjian, "Modular Self-Reconfigurable Robot Systems: Challenges and Opportunities for the Future," IEEE Robotics \& Automation Magazine, vol. 14, no. 1, pp. 43-52, 2007.

[46] S. Ahmad, H. Zhang and G. Liu, "Multiple Working Mode Control of Door-Opening With a Mobile Modular and Reconfigurable Robot," IEEE/ASME Transactions on Mechatronics, vol. 18, no. 3, pp. 833-844, 2013. 
[47] G. Liu, X. He, J. Yuan, S. Abdul and A. A. Goldenberg, "Development of Modular and Reconfigurable Robot with Multiple Working Modes," in IEEE International Conference on Robotics and Automation (ICRA), Pasadena, CA, 2008.

[48] R. C. Hibbeler, Engineering Mechanics: Dynamics, Haboken, NJ: Pearson, 2015.

[49] M. Frémond, "Collisions in Mechanics," Annals of Solids and Structural Mechanics, vol. 9, pp. 29-56, 2017.

[50] Harmonic Drive LLC, "About Harmonic Drive CSD and SHD," [Online]. Available: http://www.harmonicdrive.net/_hd/content/catalogs/pdf/csd-shd-catalog.pdf. [Accessed 1, 9 2018].

[51] C. Lelanne, Mechanical Vibration and Shock Analysis, Volume 2 - Mechanical Shock, Hoboken, NJ: John Wiley \& Sons, Inc., 2014.

[52] Harmonic Drive LLC, "General Catalog: Harmonic Drive Gearing and Motion Control," [Online]. Available:

http://www.harmonicdrive.net/_hd/content/catalogs/pdf/general_catalog_02-06_spreads.pdf. [Accessed 1, 9 2018].

[53] Harmonic Drive LLC, "Quality Certification," [Online]. Available: http://www.harmonicdrive.net/about-us/quality-certification. [Accessed 1, 9 2018].

[54] SAE Aerospace, "Aerospace Standard," [Online]. Available: http://epspinc.com/joomla/pdf/AS9100C.pdf. [Accessed 1, 9 2018].

[55] G. Liu, "Decomposition-based Friction Compensation of Mechanical Systems," Mechatronics , vol. 12, pp. 755-769, 2002.

[56] G. Liu, S. Abdul and A. A. Goldenberg, "Distributed Control of Modular and Reconfigurable Robot with Torque Sensing," Robotica, vol. 26, pp. 75-84, 2008.

[57] S. H. Lee, B.-J. Yi, S. H. Kim and Y. K. Kwak, "Modeling and Analysis of Internal Impact for General Classes of Robotic Mechanisms," in IEEE/RSJ International Conference on Intelligent Robots and Systems (IROS), Takamatsu, Japan, 2000.

[58] J. Kim, W. K. Chung and Y. Youm, "Normalized Impact Geometry and Performance Index for Redundant Manipulators," in Proc. IEEE Int. Conf. Robotics and Automation, San Francisco, CA, 2000.

[59] S. Haddadin, N. Mansfeld and A. Albu-Schaffer, "Rigid vs. Elastic Actuation: Requirements \& Performance," in IEEE/RSJ International Conference on Intelligent Robots and Systems (IROS), Vilamoura, 2012. 